Supporting Information for

\title{
Synthesis and characterization of $\left(2,6-{ }^{i} \mathrm{PrNCN}^{-} \mathrm{HfCl}_{2}{ }^{-}\right.$and $(3,5-$ $\mathrm{MeNCN})_{2} \mathrm{Hf}^{2-}$ (where $\mathrm{NCN}=2,6-$ bis[phenylazanidyl]methylphenyl): new trianionic pincer ligands.
}

\author{
Jürgen Koller, Soumya Sarkar, Khalil A. Abboud, and Adam S. Veige* \\ Department of Chemistry, Center for Catalysis, University of Florida, P.O. Box 177200 , \\ Gainesville, FL, 32611-7200
}

Index

Page

Experimental: General Considerations.

S2

Synthesis of $\left[{ }^{i} \mathrm{PrNCNLi}_{3}\right]_{2}$ (2).

S2

Synthesis of $\left[\left(2,6{ }^{i} \mathrm{PrNCN}^{-} \mathrm{HfCl}_{2}\right]\left[\mathrm{Li}(\mathrm{THF})_{2}\left(\mathrm{Et}_{2} \mathrm{O}\right)\right](\mathbf{3})\right.$.

S3

Synthesis of (3,5- $\mathrm{MeNCN}) \mathrm{H}_{2} \mathrm{Br}$ (4).

S3-S4

Synthesis of [3,5-MeNCNLi $]_{2}\left[\mathrm{Li}_{2}(\mathrm{DME})_{6}\right]$ (5).

S4

Synthesis of $\left[(3,5-\mathrm{MeNCN})_{2} \mathrm{Hf}\right]\left[\mathrm{Li}_{2}(\mathrm{DME})_{2}\right](\mathbf{6})$

S4-S5

$\mathrm{X}$-ray crystal structure solution of $\left[2,6-{ }^{i} \mathrm{PrNCNLi}_{3}\right]_{2}$ (2).

S5-S7

$\mathrm{X}$-ray crystal structure solution of $\left[\left(2,6-{ }^{i} \mathrm{PrNCN}\right) \mathrm{HfCl}_{2}\right]\left[\mathrm{Li}(\mathrm{DME})_{3}\right]$ (3-DME)

S8-S10

$\mathrm{X}$-ray crystal structure solution of $\left[3,5-\mathrm{MeNCNLi}_{2}\right]_{2}\left[\mathrm{Li}_{2}(\mathrm{DME})_{6}\right]$ (5).

S11-S14

$\mathrm{X}$-ray crystal structure solution of $\left[(3,5-\mathrm{MeNCN})_{2} \mathrm{Hf}\right]\left[\mathrm{Li}_{2}(\mathrm{DME})_{2}\right](\mathbf{6})$.

S15-S17

${ }^{1} \mathrm{H}$ and ${ }^{13} \mathrm{C}\left\{{ }^{1} \mathrm{H}\right\}$ NMR spectra of $\left[2,6-{ }^{i} \mathrm{PrNCNLi}_{3}\right]_{2}$ (2).

S18-S19

${ }^{1} \mathrm{H}$ and ${ }^{13} \mathrm{C}\left\{{ }^{1} \mathrm{H}\right\}$ NMR spectra of $\left[\left(2,6{ }^{i} \mathrm{PrNCN}\right) \mathrm{HfCl}_{2}\right]\left[\mathrm{Li}(\mathrm{THF})_{2}\left(\mathrm{Et}_{2} \mathrm{O}\right)\right](\mathbf{3})$.

$\mathrm{S} 20-\mathrm{S} 21$

${ }^{1} \mathrm{H}$ and ${ }^{13} \mathrm{C}\left\{{ }^{1} \mathrm{H}\right\}$ NMR spectra of (3,5-MeNCN) $\mathrm{H}_{2} \mathrm{Br}$ (4).

$\mathrm{S} 22-\mathrm{S} 23$

${ }^{1} \mathrm{H}$ and ${ }^{13} \mathrm{C}\left\{{ }^{1} \mathrm{H}\right\}$ NMR spectra of [3,5-MeNCNLi $]_{2}\left[\mathrm{Li}_{2}(\mathrm{DME})_{6}\right]$ (5).

S24-S25

${ }^{1} \mathrm{H}$ and ${ }^{13} \mathrm{C}\left\{{ }^{1} \mathrm{H}\right\}$ NMR spectra of $\left[(3,5-\mathrm{MeNCN})_{2} \mathrm{Hf}\right]\left[\mathrm{Li}_{2}(\mathrm{DME})_{2}\right](\mathbf{6})$

S26-S27 


\section{Experimental}

General Considerations. Unless specified otherwise, all manipulations were performed under an inert atmosphere using standard Schlenk or glovebox techniques. Pentane, hexanes, toluene, diethyl ether, tetrahydrofuran, and 1,2-dimethoxyethane were dried using a GlassContour drying column. $\mathrm{C}_{6} \mathrm{D}_{6}$ (Cambridge Isotopes) was dried over sodium-benzophenone ketyl, distilled or vacuum transferred and stored over molecular sieves. Sublimed $\mathrm{HfCl}_{4}$ was purchased from Strem Chemicals and used without further purification. MeLi, 1.6 M in diethyl ether was purchased from Acros. NMR spectra were obtained on Gemini (300 MHz), VXR (300 MHz), or Mercury (300 MHz) spectrometers. Chemical shifts are reported in $\delta(\mathrm{ppm})$. For ${ }^{1} \mathrm{H}$ and ${ }^{13} \mathrm{C}$ NMR spectra, the residual protio solvent peak was referenced as an internal reference. GC/MS spectra were recorded on an Agilent 6210 TOF-MS instrument. Elemental analyses were determined by Robertson Microlit Laboratories Inc. and Complete Analysis Laboratories Inc.

\section{Synthesis of $\left[2,6-{ }^{i} \operatorname{PrNCNLi}_{3}\right]_{2}(2)$ :}

To a solution of $1(10.0 \mathrm{~g}, 21.1 \mathrm{mmol})$ in $250 \mathrm{~mL}$ of toluene was added $46.1 \mathrm{~mL}$ of MeLi solution $(1.6 \mathrm{M}, 73.8 \mathrm{mmol})$ via syringe at $-35{ }^{\circ} \mathrm{C}$. The solution was allowed to warm to room temperature and then refluxed for $45 \mathrm{~min}$. All volatiles were removed in vacuo and the remaining solid was suspended in diethyl ether, filtered and washed with liberal amounts of diethyl ether followed by pentane to yield $\mathbf{2}$ as a white powder. The product can be recrystallized from hot toluene as colorless crystals; yield $7.28 \mathrm{~g}(15.3 \mathrm{mmol}, 70 \%) .{ }^{1} \mathrm{H} \mathrm{NMR}\left(300 \mathrm{MHz}, \mathrm{C}_{6} \mathrm{D}_{6}, \delta\right): 7.22-$ 6.91 (m, 10H, Ar Hs), 5.25 (d, $J=15 \mathrm{~Hz}, 4 \mathrm{H},-\mathrm{CH}_{2}-$ ), 4.35 (d, J=15 Hz, 4H, $-\mathrm{CH}_{2}-$ ), 3.39 (sept, $\left.J=6 \mathrm{~Hz}, 2 \mathrm{H},-\mathrm{CH}\left(\mathrm{CH}_{3}\right)_{2}\right), 2.39$ (sept, $\left.J=6 \mathrm{~Hz}, 2 \mathrm{H},-\mathrm{CH}\left(\mathrm{CH}_{3}\right)_{2}\right), 1.30$ (d, $J=6 \mathrm{~Hz}, 6 \mathrm{H},-$ $\left.\mathrm{CH}\left(\mathrm{CH}_{3}\right)_{2}\right), 1.23\left(\mathrm{~d}, J=6 \mathrm{~Hz}, 6 \mathrm{H},-\mathrm{CH}\left(\mathrm{CH}_{3}\right)_{2}\right), 0.61\left(\mathrm{~d}, J=6 \mathrm{~Hz}, 6 \mathrm{H},-\mathrm{CH}\left(\mathrm{CH}_{3}\right)_{2}\right), 0.16(\mathrm{~d}, J=6$ $\left.\mathrm{Hz}, 6 \mathrm{H},-\mathrm{CH}\left(\mathrm{CH}_{3}\right)_{2}\right) .{ }^{13} \mathrm{C}\left\{{ }^{1} \mathrm{H}\right\} \operatorname{NMR}\left(75.36 \mathrm{~Hz}, \mathrm{C}_{6} \mathrm{D}_{6}, \delta\right): 23.60\left(-\mathrm{CH}\left(\mathrm{CH}_{3}\right)_{2}\right), 24.30\left(-\mathrm{CH}\left(\mathrm{CH}_{3}\right)_{2}\right)$, $25.87\left(-\mathrm{CH}\left(\mathrm{CH}_{3}\right)_{2}\right), 27.61\left(-\mathrm{CH}\left(\mathrm{CH}_{3}\right)_{2}\right), 29.16\left(-\mathrm{CH}\left(\mathrm{CH}_{3}\right)_{2}\right), 30.36\left(-\mathrm{CH}\left(\mathrm{CH}_{3}\right)_{2}\right), 67.29\left(-\mathrm{CH}_{2}-\right)$, 122.96 (s, CH, aromatic), 124.50 (s, CH, aromatic), 125.04 (s, $\mathrm{CH}$, aromatic), 126.43 (s, CH, aromatic), 131.26 (s, CH, aromatic), 143.09 (s, CH, aromatic), 148.19 (s, CH, aromatic), 153.62 (s, $\mathrm{CH}$, aromatic), 156.45 (s, $\mathrm{N}-\mathrm{C}$, aromatic), 164.25 (br s, $\mathrm{C}-\mathrm{Li}$, aromatic). Anal. Calcd for $\mathrm{C}_{64} \mathrm{H}_{82} \mathrm{Li}_{6} \mathrm{~N}_{4}:$ C, 81.00; H, 8.71; N, 5.90. Found: C, 80.72; H, 8.44; N, 5.64. 


\section{Synthesis of $\left[\left(2,6-{ }^{i} \operatorname{PrNCN}\right) \mathrm{HfCl}_{2}\right]\left[\mathrm{Li}\left(\mathrm{THF}_{2}\left(\mathrm{Et}_{2} \mathrm{O}\right)\right](3)\right.$ :}

A suspension of $\mathrm{HfCl}_{4}(\mathrm{THF})_{2}(150 \mathrm{mg}, 323 \mu \mathrm{mol})$ in $5 \mathrm{~mL}$ of $\mathrm{THF}$ was cooled to $-35^{\circ} \mathrm{C}$ and added dropwise to a solution of $2(153 \mathrm{mg}, 161 \mu \mathrm{mol})$ in $5 \mathrm{~mL}$ of THF at $-35^{\circ} \mathrm{C}$. The solution was allowed to warm to room temperature and stirred for an additional $60 \mathrm{~min}$. The solvent was removed in vacuo and the remaining solid was extracted with diethyl ether $(2 \times 5 \mathrm{~mL})$. The organic layers were combined, filtered, concentrated and then recrystallized at $-35^{\circ} \mathrm{C}$. The product 3 was

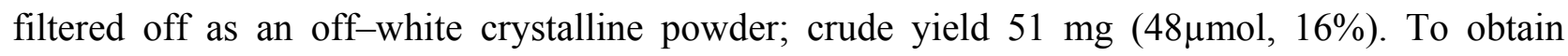
analytically pure samples, the crude product can be recrystallized multiple times from a saturated diethyl ether solution at $-35{ }^{\circ} \mathrm{C}$. After multiple recrystallizations THF solvent molecules were replaced with diethyl ether. ${ }^{1} \mathrm{H}$ NMR $\left(300 \mathrm{MHz}, \mathrm{C}_{6} \mathrm{D}_{6}, \delta\right): 7.22-6.91$ (m, 10H, Ar Hs), 5.56 (s, 4H, $-\mathrm{CH}_{2}-$ ), 4.00 (sept, $\left.J=6 \mathrm{~Hz}, 4 \mathrm{H},-\mathrm{CH}\left(\mathrm{CH}_{3}\right)_{2}\right), 3.10$, (q, $J=6 \mathrm{~Hz}, 4 \mathrm{H}_{,} \mathrm{Et}_{2} \mathrm{O} /-\mathrm{OCH}_{2}-$, overlapping) 3.07 (br s, 8H, THF/-OCH $2_{2}^{-}$, orverlapping), 1.49 (d, $\left.J=6 \mathrm{~Hz}, 12 \mathrm{H},-\mathrm{CH}\left(\mathrm{CH}_{3}\right)_{2}\right), 1.25$ (d, $J=6$ $\mathrm{Hz}, 12 \mathrm{H},-\mathrm{CH}\left(\mathrm{CH}_{3}\right)_{2}$, overlapping), 1.24 (br s, 8H, THF/- $\mathrm{CH}_{2}-$, overlapping), 0.96 (t, $\mathrm{J}=6 \mathrm{~Hz}$, $\left.6 \mathrm{H}, \mathrm{Et}_{2} \mathrm{O} /-\mathrm{CH}_{3}\right) .{ }^{13} \mathrm{C}\left\{{ }^{1} \mathrm{H}\right\} \operatorname{NMR}\left(75.36 \mathrm{MHz}, \mathrm{C}_{6} \mathrm{D}_{6}, \delta\right): 15.45\left(\mathrm{~s}, \mathrm{Et}_{2} \mathrm{O} /-\mathrm{OCH}_{3}\right), 24.74$ (s, $\left.\mathrm{CH}\left(\mathrm{CH}_{3}\right)_{2}\right), 25.82\left(\mathrm{~s}, \mathrm{THF} /-\mathrm{CH}_{2}-\right), 28.32\left(\mathrm{~s},-\mathrm{CH}\left(\mathrm{CH}_{3}\right)_{2}\right), 28.7\left(\mathrm{~s},-\mathrm{CH}\left(\mathrm{CH}_{3}\right)_{2}\right), 66.09(\mathrm{THF} /-$ $\left.\mathrm{OCH}_{2}\right), 69.33\left(\mathrm{~s}, \mathrm{Et}_{2} \mathrm{O} /-\mathrm{OCH}_{2}-\right), 73.74\left(\mathrm{~s},-\mathrm{CH}_{2}-\right), 119.04\left(\mathrm{~s}, \mathrm{CH}\right.$, aromatic), $124.33\left(\mathrm{~s}, \mathrm{C}\left({ }^{i} \mathrm{Pr}\right) \mathrm{CH}\right.$, aromatic), 124.99 (s, $\mathrm{CH}$, aromatic), 127.52 (s, $\mathrm{C}\left({ }^{i} \mathrm{Pr}\right) \mathrm{CHCH}$, aromatic), $148.44\left(\mathrm{~s}, \mathrm{C}\left(\mathrm{CH}_{2}\right) \mathrm{CH}\right.$, aromatic), $151.85\left(\mathrm{~s}, \mathrm{C}\left(\mathrm{CH}_{2}\right) \mathrm{CHCH}\right.$, aromatic), 156.07 (s, $\mathrm{N}-\mathrm{C}$, aromatic, 201.55 (s, $\mathrm{Hf}-\mathrm{C}$, aromatic). Anal. Calcd for $\mathrm{C}_{44} \mathrm{H}_{71} \mathrm{Cl}_{2} \mathrm{HfLiN}_{2} \mathrm{O}_{6}: \mathrm{C}, 53.90 ; \mathrm{H}, 7.30 ; \mathrm{N}, 2.86$. Found: C, 53.84; $\mathrm{H}$, $6.63 ; \mathrm{N}, 3.00$.

\section{Synthesis of 3,5-MeNCNH $2 \mathrm{Br}(4)$ :}

To a solution of 3,5-dimethlyaniline, $(8.26 \mathrm{~mL}, 66 \mathrm{mmol})$ in $125 \mathrm{~mL}$ of THF, was added $26.7 \mathrm{~mL}$ of ${ }^{n} \mathrm{BuLi}$ solution $(2.5 \mathrm{M}$ in hexanes, $66 \mathrm{mmol})$ via syringe at $0{ }^{\circ} \mathrm{C}$. The solution was allowed to warm to room temperature and then 2-bromo-1,3-bis(bromomethyl)benzene (11.4 g, $33 \mathrm{mmol})$ was added as a THF $(30 \mathrm{~mL})$ solution. The solution quickly turned dark brown and was refluxed for $20 \mathrm{~h}$. After removing all volatiles the resulting brown oil was taken up in diethyl ether and washed with $\mathrm{H}_{2} \mathrm{O}(2 \times 10 \mathrm{~mL})$. The organic layer was dried over $\mathrm{MgSO}_{4}$, filtered, and then evaporated. The resulting light brown solid was recrystallized from hot hexanes to provide $\mathbf{3}$ as a tan microcrystalline solid; yield $7.31 \mathrm{~g}$ (17.3 mmol, 52\%). ${ }^{1} \mathrm{H}$ NMR $\left(300 \mathrm{MHz}, \mathrm{C}_{6} \mathrm{D}_{6}, \delta\right): 7.14(\mathrm{~d}, J$

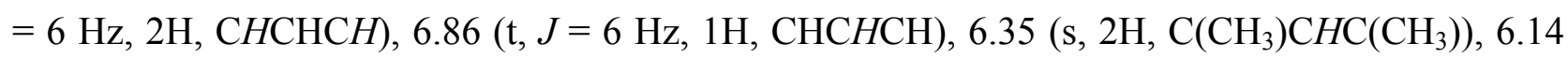


(s, 4H, N-CCH), 4.25 (d, $\left.J=6 \mathrm{~Hz}, 4 \mathrm{H},-\mathrm{CH}_{2}-\right), 3.52$ (t, $\left.J=6 \mathrm{~Hz}, \mathrm{NH}\right), 2.10$ (s, 12H, $-\mathrm{CH}_{3}$ ). ${ }^{13} \mathrm{C}\left\{{ }^{1} \mathrm{H}\right\} \quad \mathrm{NMR} \quad\left(75.36, \quad \mathrm{C}_{6} \mathrm{D}_{6}, \quad \delta\right): \quad 22.02 \quad\left(\mathrm{~s}, \quad \mathrm{CH}_{3}\right), \quad 49.28 \quad\left(\mathrm{~s}, \quad-\mathrm{CH}_{2}-\right), 111.53 \quad(\mathrm{~s}$, $\mathrm{CHC}\left(\mathrm{CH}_{3}\right) \mathrm{CHC}\left(\mathrm{CH}_{3}\right) \mathrm{CH}$, aromatic), 120.49 (s, $\mathrm{CHC}\left(\mathrm{CH}_{3}\right) \mathrm{CHC}\left(\mathrm{CH}_{3}\right) \mathrm{CH}$, aromatic), 124.37 (s, C-Br, aromatic), 127.92 (s, $\mathrm{CHCHCH}$, aromatic), 128.33 (s, $\mathrm{CHCHCH}$, aromatic), 139.17 (s, $\mathrm{CHC}\left(\mathrm{CH}_{3}\right) \mathrm{CHC}\left(\mathrm{CH}_{3}\right) \mathrm{CH}$, aromatic), 139.94 (s, $\mathrm{CCHCH}$, aromatic), 148.61 (s, N-C, aromatic). ESI-MS $(m / z):[\mathrm{M}+\mathrm{H}]^{+}$calcd for $\mathrm{C}_{24} \mathrm{H}_{27} \mathrm{BrN}_{2}$, 423.144; found, 423.142. Anal. Calcd for $\mathrm{C}_{24} \mathrm{H}_{27} \mathrm{BrN}_{2}$ : C, 68.08; H, 6.43; N, 6.62. Found: C, 67.92; H, 6.61; N, 6.62 .

\section{Synthesis of $\left[3,5-\mathrm{MeNCNLi}_{2}\right]_{2}\left[\mathrm{Li}_{2}(\mathrm{DME})_{6}\right](5)$ :}

To an orange DME $(3 \mathrm{~mL})$ solution of $4(500 \mathrm{mg}, 1.18 \mathrm{mmol})$ was added 3.3 equiv of ${ }^{n} \mathrm{BuLi}$ solution $(1.56 \mathrm{~mL}, 2.5 \mathrm{M}$ in hexanes, $3.89 \mathrm{mmol})$ via syringe at $-35^{\circ} \mathrm{C}$. Upon warming to room temperature a bright yellow precipitate formed. After stirring for $5 \mathrm{~min}$ the solution was filtered to provide a yellow solid that was washed with DME then dried in vacuo to provide $\mathbf{5}$ as a yellow microcrystalline solid; yield $366 \mathrm{mg}(0.58 \mathrm{mmol}, 50 \%$. The yellow salt 5 can be further

purified by recrystallization from hot benzene in necessary. ${ }^{1} \mathrm{H} N \mathrm{NM}\left(300 \mathrm{MHz}, \mathrm{C}_{6} \mathrm{D}_{6}, \delta\right): 7.50$ (s, 4H, $\mathrm{CHCHCH}$ ), 7.50 (s, 2H, $\mathrm{CHCHCH}), 6.08$ (br s, 4H, $\left.\mathrm{C}\left(\mathrm{CH}_{3}\right) \mathrm{CHC}\left(\mathrm{CH}_{3}\right)\right), 6.06(\mathrm{~s}, 8 \mathrm{H}, \mathrm{N}-$ $\mathrm{CCH}$ ), 4.78 (s, $8 \mathrm{H},-\mathrm{CH}_{2}-$ ), 2.90 (br s, 36H, DME/-OCH $H_{3}$ ), 2.83 (br s, 24H, DME/-CH $\mathrm{C}_{2} \mathrm{O}$ ), 2.19 (s, $\left.24 \mathrm{H},-\mathrm{CH}_{3}\right) .{ }^{13} \mathrm{C}\left\{{ }^{1} \mathrm{H}\right\}$ NMR (75.36, toluene-d $\left.8, \delta\right): 22.14\left(\mathrm{~s}, \mathrm{CH}_{3}\right), 58.98\left(\mathrm{~s}, \mathrm{DME} /-\mathrm{OCH}_{2}\right)$, 60.89 (s, $-\mathrm{CH}_{2}-$ ), 70.95 (s, DME/-OCH ${ }_{3}$ ), 111.92 (br s, $C$ - Li, aromatic), 113.56 (s, $C \mathrm{HCHCH}$, aromatic), 123.80 (s, $\mathrm{CHC}\left(\mathrm{CH}_{3}\right) \mathrm{CHC}\left(\mathrm{CH}_{3}\right) \mathrm{CH}$, aromatic), 126.18 (s, $\mathrm{CHCHCH}$, aromatic), 138.05 (s, $\mathrm{CHC}\left(\mathrm{CH}_{3}\right) \mathrm{CHC}\left(\mathrm{CH}_{3}\right) \mathrm{CH}$, aromatic), 142.43 (s, $\mathrm{CHC}\left(\mathrm{CH}_{3}\right) \mathrm{CHC}\left(\mathrm{CH}_{3}\right) \mathrm{CH}$, aromatic), 156.99 (s, $C \mathrm{CHCH}$, aromatic), 160.54 (s, $\mathrm{N}-\mathrm{C}$, aromatic). Anal. Calcd for $\mathrm{C}_{72} \mathrm{H}_{110} \mathrm{HfLi}_{6} \mathrm{~N}_{4} \mathrm{O}_{12}: \mathrm{C}, 68.34 ; \mathrm{H}$, 8.76; N, 4.43. Found: C, 68.12; H, 8.49; N, 4.55 .

\section{Synthesis of $\left[(3,5-\mathrm{MeNCN})_{2} \mathrm{Hf}\right]\left[\mathrm{Li}_{2}(\mathrm{DME})_{2}\right](6)$ :}

Benzene solutions of $5(300 \mathrm{mg}, 0.24 \mathrm{mmol})$ and $\mathrm{HfCl}_{4}(76 \mathrm{mg}, 0.24 \mathrm{mmol})$ were combined at room temperature. The initial suspension quickly dissolved and then a white precipitate formed benzene which was removed by filtration. The product was precipitated as a white microcrystalline solid by addition of the filtrate $(5 \mathrm{~mL})$ to $100 \mathrm{~mL}$ of cold pentane; yield 137 mg (0.13 mmol, 54\%). ${ }^{1} \mathrm{H}$ NMR (300 MHz, $\left.\mathrm{C}_{6} \mathrm{D}_{6}, \delta\right): 7.45$ (s, 4H, CHCHCH), 7.45 (s, 2H, $\mathrm{CHCHCH}), 7.01(\mathrm{~s}, 8 \mathrm{H}, \mathrm{N}-\mathrm{CCH}), 6.16\left(\mathrm{~s}, 4 \mathrm{H}, \mathrm{C}\left(\mathrm{CH}_{3}\right) \mathrm{CHC}\left(\mathrm{CH}_{3}\right), 5.56\left(\mathrm{~s}, 8 \mathrm{H},-\mathrm{CH}_{2}-\right), 2.89\right.$ (br s, 
12H, DME/-OCH $H_{3}$ ), 2.78 (br s, 8H, DME/-C $\mathrm{CH}_{2} \mathrm{O}$ ), 2.00 (s, 24H, $\left.-\mathrm{CH}_{3}\right) .{ }^{13} \mathrm{C}\left\{{ }^{1} \mathrm{H}\right\}$ NMR (75.36, $\mathrm{C}_{6} \mathrm{D}_{6}, \delta$ ): $21.97\left(\mathrm{~s}, \mathrm{CH}_{3}\right), 59.28$ (s, DME/-OCH $\left.{ }_{2}\right), 69.26$ (s, $\left.-\mathrm{CH}_{2}-\right), 71.48$ (s, DME/-OCH ${ }_{3}$ ), 117.01 (s, $\mathrm{CHC}\left(\mathrm{CH}_{3}\right) \mathrm{CHC}\left(\mathrm{CH}_{3}\right) \mathrm{CH}$, aromatic), 118.76 (s, $\mathrm{CCHCH}$, aromatic), 119.68 (s, $\mathrm{CHC}\left(\mathrm{CH}_{3}\right) \mathrm{CHC}\left(\mathrm{CH}_{3}\right) \mathrm{CH}$, aromatic), 127.63 (s, N-C, aromatic), 137.66 (s, $\mathrm{CHC}\left(\mathrm{CH}_{3}\right) \mathrm{CH}\left(\mathrm{CH}_{3}\right) \mathrm{CH}$, aromatic), 154.83 (s, $\mathrm{CHCHCH}$, aromatic), 156.94 (s, $\mathrm{CHCHCH}$, aromatic), 204.28 (s, Hf-C, aromatic). Anal. Calcd for $\mathrm{C}_{56} \mathrm{H}_{70} \mathrm{HfLi}_{2} \mathrm{~N}_{4} \mathrm{O}_{4}: \mathrm{C}, 63.72 ; \mathrm{H}, 6.68 ; \mathrm{N}$, 5.31. Found: C, 61.69; H, 6.52; N, 5.31.

\section{$\mathrm{X}$-ray experimental details for $\left[2,6-{ }^{i} \operatorname{PrNCNLi}_{3}\right]_{2}(2)$ :}

Data were collected at $173 \mathrm{~K}$ on a Siemens SMART PLATFORM equipped with A CCD area detector and a graphite monochromator utilizing $\operatorname{MoK}_{\alpha}$ radiation $(\lambda=0.71073 \AA)$. Cell parameters were refined using up to 8192 reflections. A full sphere of data (1850 frames) was collected using the $\omega$-scan method $\left(0.3^{\circ}\right.$ frame width). The first 50 frames were re-measured at the end of data collection to monitor instrument and crystal stability (maximum correction on I was $<1 \%$ ). Absorption corrections by integration were applied based on measured indexed crystal faces.

The structure was solved by the Direct Methods in SHELXTL6, and refined using fullmatrix least squares. The non-H atoms were treated anisotropically, whereas the hydrogen atoms were calculated in ideal positions and were riding on their respective carbon atoms. The asymmetric unit consists of two anions ( -3$)$ and six $\mathrm{Li}+$ cations. The six $\mathrm{Li}$ ions are surrounded by the two anions where each one of them interacts with two Nitrogen atoms of the anions. The Difference Fourier map was examined to decide if there were protons on $\mathrm{Cl}$ and $\mathrm{Cla}$ but the results were negative. Additionally, there does not seem to be enough space for the existence of protons on the above mentioned atoms. Data was truncated at a maximum $2 \Theta=45^{\circ}$ due to small crystal size which produced an average intensity of $0.29 \sigma$. A total of 664 parameters were refined in the final cycle of refinement using 19711 reflections with $\mathrm{I}>2 \sigma$ (I) to yield $\mathrm{R}_{1}$ and $\mathrm{wR}_{2}$ of $5.89 \%$ and $9.16 \%$, respectively. Refinement was done using $\mathrm{F}^{2}$. 
Table S1. Crystal data, structure solution and refinement for $\left[2,6-{ }^{i} \mathrm{PrNCNLi}_{3}\right]_{2}$ (2).

\begin{tabular}{|c|c|}
\hline identification code & $\mathrm{kv01}$ \\
\hline empirical formula & $\mathrm{C}_{32} \mathrm{H}_{41} \mathrm{Li}_{3} \mathrm{~N}_{2}$ \\
\hline formula weight & 474.49 \\
\hline$T(\mathrm{~K})$ & $173(2)$ \\
\hline$\lambda(\AA)$ & 0.71073 \\
\hline crystal system & Orthorhombic \\
\hline space group & Pbca \\
\hline$a(\AA)$ & $20.091(3)$ \\
\hline$b(\AA)$ & $19.288(2)$ \\
\hline$c(\AA)$ & $29.998(4)$ \\
\hline$\alpha(\operatorname{deg})$ & 90 \\
\hline$\beta(\operatorname{deg})$ & 90 \\
\hline$\gamma(\operatorname{deg})$ & 90 \\
\hline$V\left(\AA^{3}\right)$ & $11625(3)$ \\
\hline$Z$ & 16 \\
\hline$\rho_{\text {calcd }}\left(\mathrm{Mg} \mathrm{mm}^{-3}\right)$ & 1.084 \\
\hline crystal size $\left(\mathrm{mm}^{3}\right)$ & $0.26 \times 0.09 \times 0.04$ \\
\hline abs coeff $\left(\mathrm{mm}^{-1}\right)$ & 0.061 \\
\hline$F(000)$ & 4096 \\
\hline$\theta$ range for data collection & 1.36 to 22.50 \\
\hline limiting indices & $-19 \leq h \leq 19,-15 \leq k \leq 19,-32 \leq \ell \leq 5$ \\
\hline no. of reflns collcd & 19711 \\
\hline no. of ind reflns $\left(R_{\text {int }}\right)$ & $6061(0.1904)$ \\
\hline completeness to $\theta=23.25^{\circ}$ & $79.8 \%$ \\
\hline absorption corr & Integration \\
\hline refinement method & Full-matrix least-squares on $\mathrm{F}^{2}$ \\
\hline data / restraints / parameters & $6061 / 0 / 664$ \\
\hline$R 1,{ }^{\mathrm{a}} w R 2^{\mathrm{b}}[\mathrm{I}>2 \sigma]$ & $0.0589,0.0916$ \\
\hline$R 1,{ }^{\mathrm{a}} w R 2^{\mathrm{b}}$ (all data) & $0.1887,0.1242$ \\
\hline $\mathrm{GOF}^{\mathrm{c}}$ on $F^{2}$ & 0.874 \\
\hline largest diff. peak and hole & 0.182 and $-0.152 \mathrm{e}^{-3}$ \\
\hline
\end{tabular}




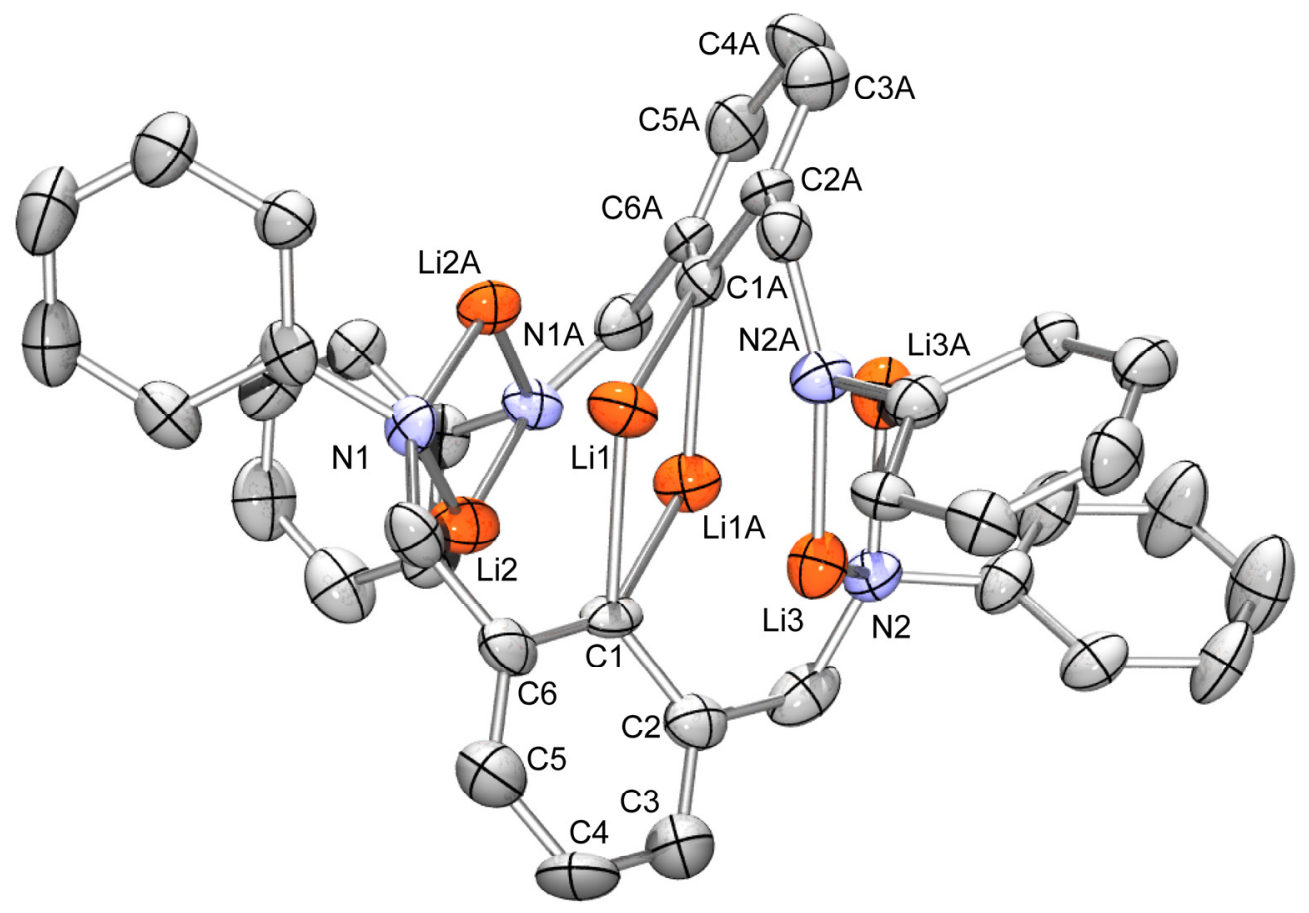

Figure S1. Molecular Structure of $\left[2,6-{ }^{i} \mathrm{PrNCNLi}_{3}\right]_{2}(2)$ with ellipsoids drawn at the $50 \%$ probability level. Hydrogen atoms and isopropyl groups have been omitted for clarity. 


\section{$\mathrm{X}$-ray experimental details for $\left[\left(2,6{ }^{i}{ }^{i} \mathrm{PrNCN}\right) \mathrm{HfCl}_{2}\right]\left[\mathrm{Li}(\mathrm{DME})_{3}\right]$ (3-DME):}

Data were collected at $173 \mathrm{~K}$ on a Siemens SMART PLATFORM equipped with A CCD area detector and a graphite monochromator utilizing $\operatorname{MoK}_{\alpha}$ radiation $(\lambda=0.71073 \AA)$. Cell parameters were refined using up to 8192 reflections. A full sphere of data (1850 frames) was collected using the $\omega$-scan method $\left(0.3^{\circ}\right.$ frame width). The first 50 frames were re-measured at the end of data collection to monitor instrument and crystal stability (maximum correction on I was $<1 \%$ ). Absorption corrections by integration were applied based on measured indexed crystal faces. The structure was solved by the Direct Methods in SHELXTL6, and refined using full-matrix least squares. The non- $\mathrm{H}$ atoms were treated anisotropically, whereas the hydrogen atoms were calculated in ideal positions and were riding on their respective carbon atoms. The asymmetric unit consists of the Hf complex anion and L+ surrounded by three DME molecules. Two of the latter are disordered and were refined in two parts with their site occupation factors dependently refined. The disordered DME were constrained to maintain the same geometry as that of the third DME. All atoms of the disordered parts were refined with isotropic displacement parameters. A total of 494 parameters were refined in the final cycle of refinement using 31825 reflections with $\mathrm{I}>2 \sigma(\mathrm{I})$ to yield $\mathrm{R}_{1}$ and $\mathrm{wR}_{2}$ of $6.99 \%$ and $14.02 \%$, respectively. Refinement was done using $\mathrm{F}^{2}$. 
Table S2. Crystal data, structure solution and refinement for $[(2,6-$ $\left.\left.{ }^{i} \mathrm{PrNCN}\right) \mathrm{HfCl}_{2}\right]\left[\mathrm{Li}(\mathrm{DME})_{3}\right]$ (3-DME).

\begin{tabular}{|c|c|}
\hline identification code & kv26 \\
\hline empirical formula & $\mathrm{C}_{44} \mathrm{H}_{71} \mathrm{LiN}_{2} \mathrm{O}_{6} \mathrm{Cl}_{2} \mathrm{Hf}$ \\
\hline formula weight & 980.36 \\
\hline$T(\mathrm{~K})$ & $173(2)$ \\
\hline$\lambda(\AA)$ & 0.71073 \\
\hline crystal system & Monoclinic \\
\hline space group & $\mathrm{P} 2{ }_{1} / \mathrm{c}$ \\
\hline$a(\AA)$ & $14.2626(11)$ \\
\hline$b(\AA)$ & $12.6991(2)$ \\
\hline$c(\AA)$ & $27.443(2)$ \\
\hline$\alpha(\mathrm{deg})$ & 90 \\
\hline$\beta(\operatorname{deg})$ & $98.627(2)$ \\
\hline$\gamma(\operatorname{deg})$ & 90 \\
\hline$V\left(\AA^{3}\right)$ & $4914.3(7)$ \\
\hline$Z$ & 4 \\
\hline$\rho_{\text {calcd }}\left(\mathrm{Mg} \mathrm{mm}^{-3}\right)$ & 1.325 \\
\hline crystal size $\left(\mathrm{mm}^{3}\right)$ & $0.09 \times 0.09 \times 0.02$ \\
\hline abs coeff $\left(\mathrm{mm}^{-1}\right)$ & 2.274 \\
\hline$F(000)$ & 2024 \\
\hline$\theta$ range for data collection & 1.50 to 27.50 \\
\hline limiting indices & $-18 \leq h \leq 17,-16 \leq k \leq 15,-35 \leq \ell \leq 17$ \\
\hline no. of reflns collcd & 31825 \\
\hline no. of ind reflns $\left(R_{\text {int }}\right)$ & $11123(0.1139)$ \\
\hline completeness to $\theta=23.25^{\circ}$ & $98.5 \%$ \\
\hline absorption corr & Integration \\
\hline refinement method & Full-matrix least-squares on $\mathrm{F}^{2}$ \\
\hline data / restraints / parameters & $11123 / 100 / 490$ \\
\hline$R 1,{ }^{\mathrm{a}} w R 2^{\mathrm{b}}[\mathrm{I}>2 \sigma]$ & $0.0704,0.1418$ \\
\hline$R 1,{ }^{\mathrm{a}} w R 2^{\mathrm{b}}$ (all data) & $0.1439,0.1669$ \\
\hline $\mathrm{GOF}^{\mathrm{c}}$ on $F^{2}$ & 1.024 \\
\hline largest diff. peak and hole & 1.398 and $-1.283 \mathrm{e} \AA^{-3}$ \\
\hline \multicolumn{2}{|l|}{$\mathrm{R} 1=\sum\left(|| \mathrm{F}_{\mathrm{O}}|-| \mathrm{F}_{\mathrm{c}}||\right) / \sum\left|\mathrm{F}_{\mathrm{O}}\right|$} \\
\hline \multicolumn{2}{|c|}{$\mathrm{wR} 2=\left[\Sigma\left[\mathrm{w}\left(\mathrm{Fo}_{\mathrm{o}}^{2}-\mathrm{F}^{2}\right)^{2}\right] / \Sigma\left[\mathrm{w}\left(\mathrm{F}_{\mathrm{o}}^{2}\right)^{2}\right]\right]^{1 / 2}$} \\
\hline \multicolumn{2}{|c|}{$\mathrm{S}=\left[\Sigma\left[\mathrm{w}\left(\mathrm{F}_{\mathrm{o}}^{2}-\mathrm{F}_{\mathrm{c}}^{2}\right)^{2}\right] /(\mathrm{n}-\mathrm{p})\right]^{1 / 2}$} \\
\hline
\end{tabular}




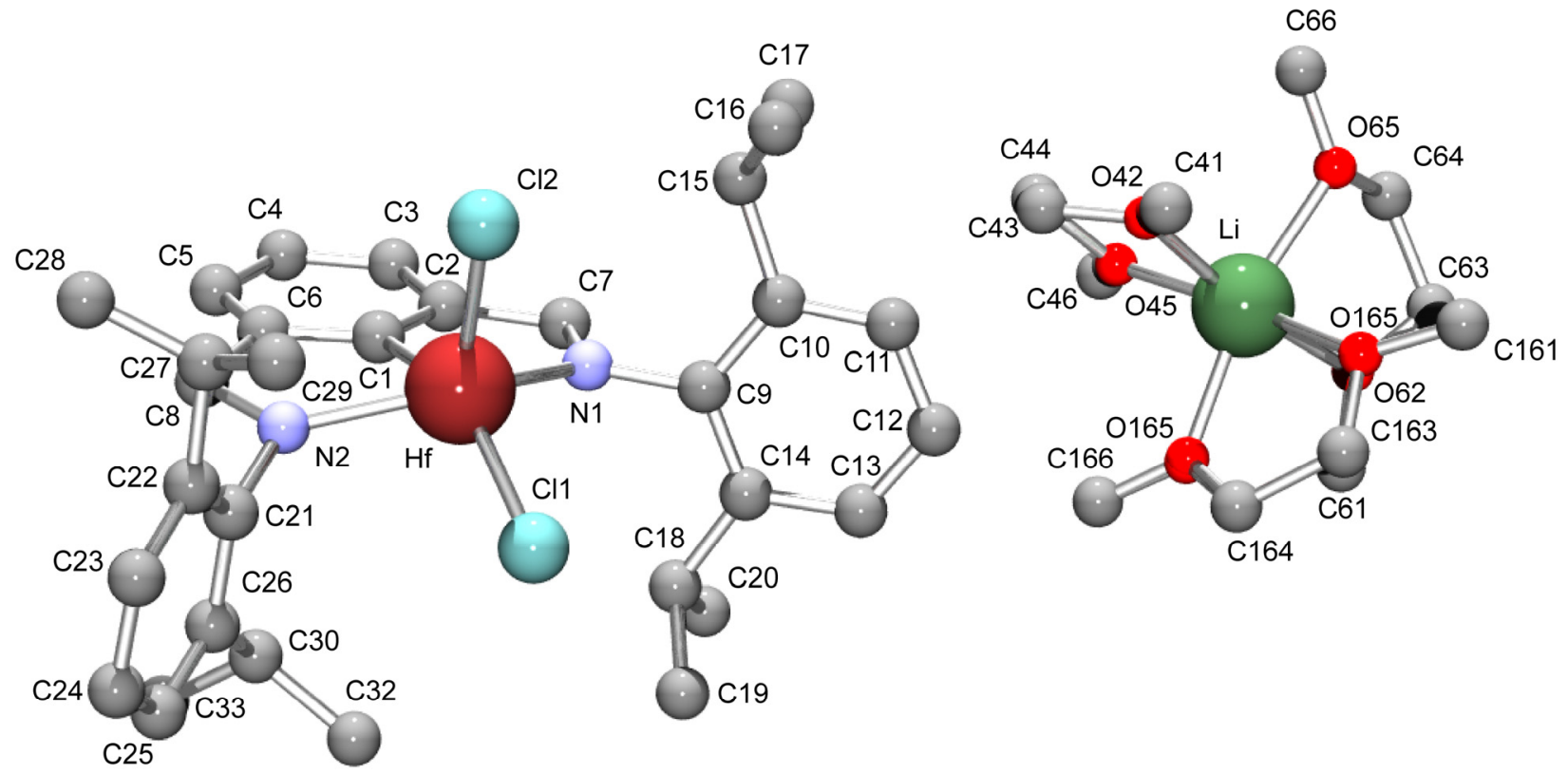

Figure S2. Molecular Structure of $\left[\left(2,6-{ }^{i} \mathrm{PrNCN} \mathrm{HfCl}_{2}\right]\left[\mathrm{Li}(\mathrm{DME})_{3}\right]\right.$ (3-DME). Hydrogen atoms are omitted for clarity. 


\section{$\mathrm{X}$-ray experimental details for $\left[3,5-\mathrm{MeNCNLi}_{2}\right]_{2} \operatorname{Li}_{2}(\mathrm{DME})_{6}(5)$ :}

Data were collected at $173 \mathrm{~K}$ on a Siemens SMART PLATFORM equipped with A CCD area detector and a graphite monochromator utilizing Mo $\mathrm{K}_{\alpha}$ radiation $(\lambda=0.71073 \AA)$. Cell parameters were refined using up to 8192 reflections. A full sphere of data (1850 frames) was collected using the $\omega$-scan method $\left(0.3^{\circ}\right.$ frame width $)$. The first 50 frames were re-measured at the end of data collection to monitor instrument and crystal stability (maximum correction on I was $<1 \%$ ). Absorption corrections by integration were applied based on measured indexed crystal faces.

The structure was solved by the Direct Methods in SHELXTL6, and refined using fullmatrix least squares. The non-H atoms were treated anisotropically, whereas the hydrogen atoms were calculated in ideal positions and were riding on their respective carbon atoms. The structure consists of a core of $\left.\mathrm{Li}_{4}\right]^{+4}$ (located on -4 symmetry elements) coordinated to two triply deprotonated ligands $\left.\mathrm{L}_{2}\right]^{-6}$, and $\mathrm{Li}(\mathrm{DME})_{3}{ }^{+1}$ cations located on two-fold rotation axes along the z-axis. Thus the asymmetric unit consists of a $1 / 4$ cation and a half anion. The counter cation is fully disordered as a result of sitting on two-fold rotation symmetry, thus all atoms are refined with 50\% occupancy. Two DME ligands were constrained during refinement to geometries similar to the third, well behaved, DME ligand. A total of 277 parameters were refined in the final cycle of refinement using 2745 reflections with $\mathrm{I}>2 \sigma(\mathrm{I})$ to yield $\mathrm{R}_{1}$ and $\mathrm{wR}_{2}$ of $4.93 \%$ and $12.21 \%$, respectively. Refinement was done using $\mathrm{F}^{2}$. 
Table S2. Crystal data, structure solution and refinement for [3,5-MeNCNLi $\left.]_{2}\right]_{2}\left[\mathrm{Li}_{2}(\mathrm{DME})_{6}\right]$ (5).

\begin{tabular}{|c|c|}
\hline identification code & ss05 \\
\hline empirical formula & $\mathrm{C}_{72} \mathrm{H}_{110} \mathrm{Li}_{6} \mathrm{~N}_{4} \mathrm{O}_{12}$ \\
\hline formula weight & 1265.28 \\
\hline$T(\mathrm{~K})$ & $173(2)$ \\
\hline$\lambda(\AA)$ & 0.71073 \\
\hline crystal system & Tetragonal \\
\hline space group & $\mathrm{P}-42{ }_{1} \mathrm{c}$ \\
\hline$a(\AA)$ & $15.3065(5)$ \\
\hline$b(\AA)$ & $15.3065(5)$ \\
\hline$c(\AA)$ & $16.118(1)$ \\
\hline$\alpha(\mathrm{deg})$ & 90 \\
\hline$\beta(\operatorname{deg})$ & 90 \\
\hline$\gamma(\operatorname{deg})$ & 90 \\
\hline$V\left(\AA^{3}\right)$ & $3776.3(3)$ \\
\hline$Z$ & 2 \\
\hline$\rho_{\text {calcd }}\left(\mathrm{Mg} \mathrm{mm}^{-3}\right)$ & 1.113 \\
\hline crystal size $\left(\mathrm{mm}^{3}\right)$ & $0.34 \times 0.20 \times 0.11$ \\
\hline abs coeff $\left(\mathrm{mm}^{-1}\right)$ & 0.073 \\
\hline$F(000)$ & 1368 \\
\hline$\theta$ range for data collection & 1.83 to 27.50 \\
\hline limiting indices & $-19 \leq h \leq 19,-12 \leq k \leq 19,-20 \leq \ell \leq 17$ \\
\hline no. of reflns collcd & 24967 \\
\hline no. of ind reflns $\left(R_{\text {int }}\right)$ & $4331(0.0853)$ \\
\hline completeness to $\theta=23.25^{\circ}$ & $100.0 \%$ \\
\hline absorption corr & None \\
\hline refinement method & Full-matrix least-squares on $\mathrm{F}^{2}$ \\
\hline data / restraints / parameters & $4331 / 0 / 277$ \\
\hline$R 1^{\mathrm{a}}{ }^{\mathrm{a}} w R 2^{\mathrm{b}}[\mathrm{I}>2 \sigma]$ & $0.0493,0.1221$ \\
\hline$R 1,{ }^{\mathrm{a}} w R 2^{\mathrm{b}}$ (all data) & $0.0860,0.1384$ \\
\hline $\mathrm{GOF}^{\mathrm{c}}$ on $F^{2}$ & 0.943 \\
\hline largest diff. peak and hole & 0.154 and $-0.151 \mathrm{e}^{-3}$ \\
\hline
\end{tabular}




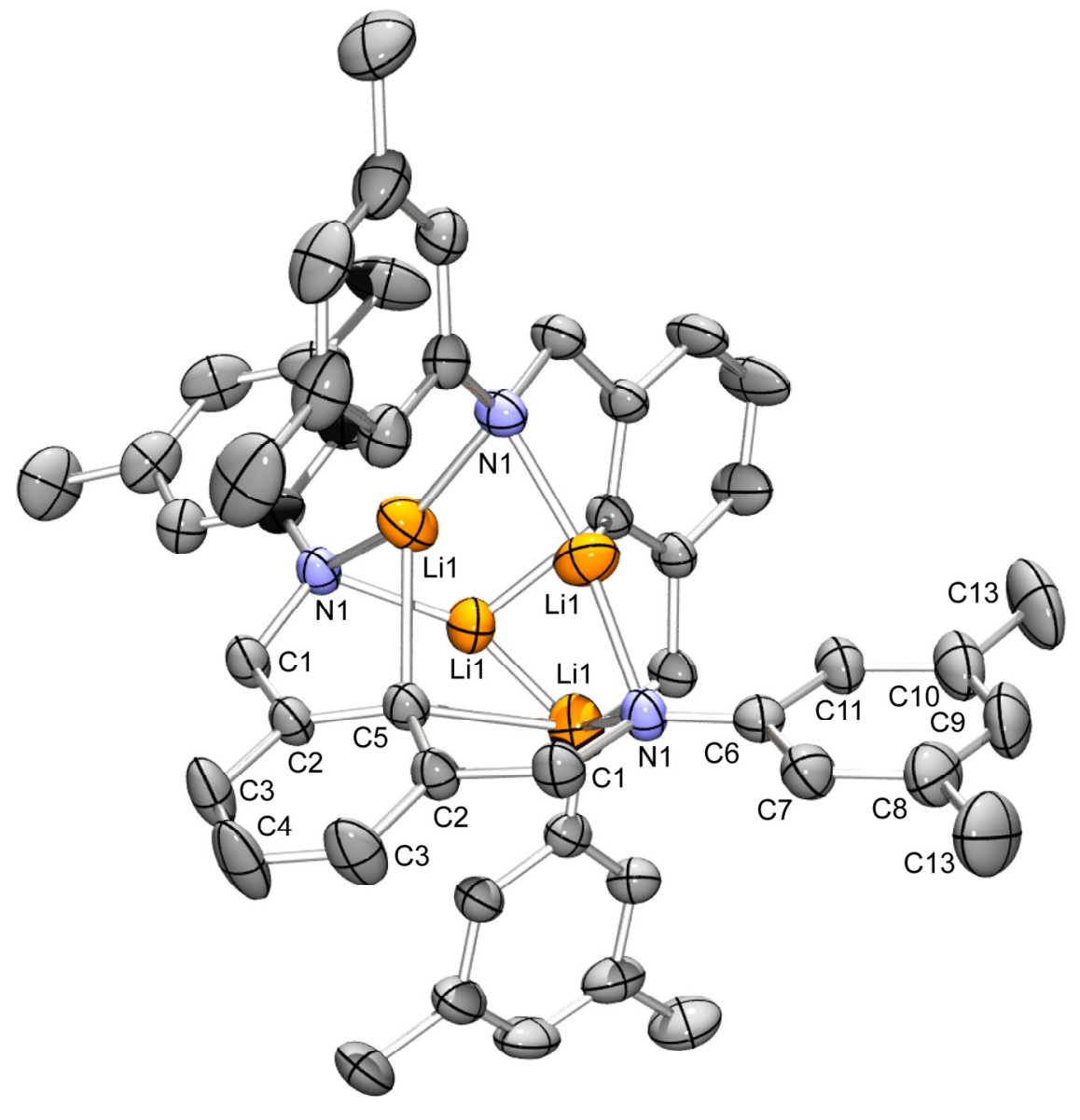

Figure S3. Molecular Structure of [3,5- $\left.\mathrm{MeNCNLi}_{2}\right]_{2}\left[\mathrm{Li}_{2}(\mathrm{DME})_{6}\right](\mathbf{5})$ with ellipsoids drawn at the $50 \%$ probability level. Hydrogen atoms, lithium counter ions and DME omitted for clarity. 

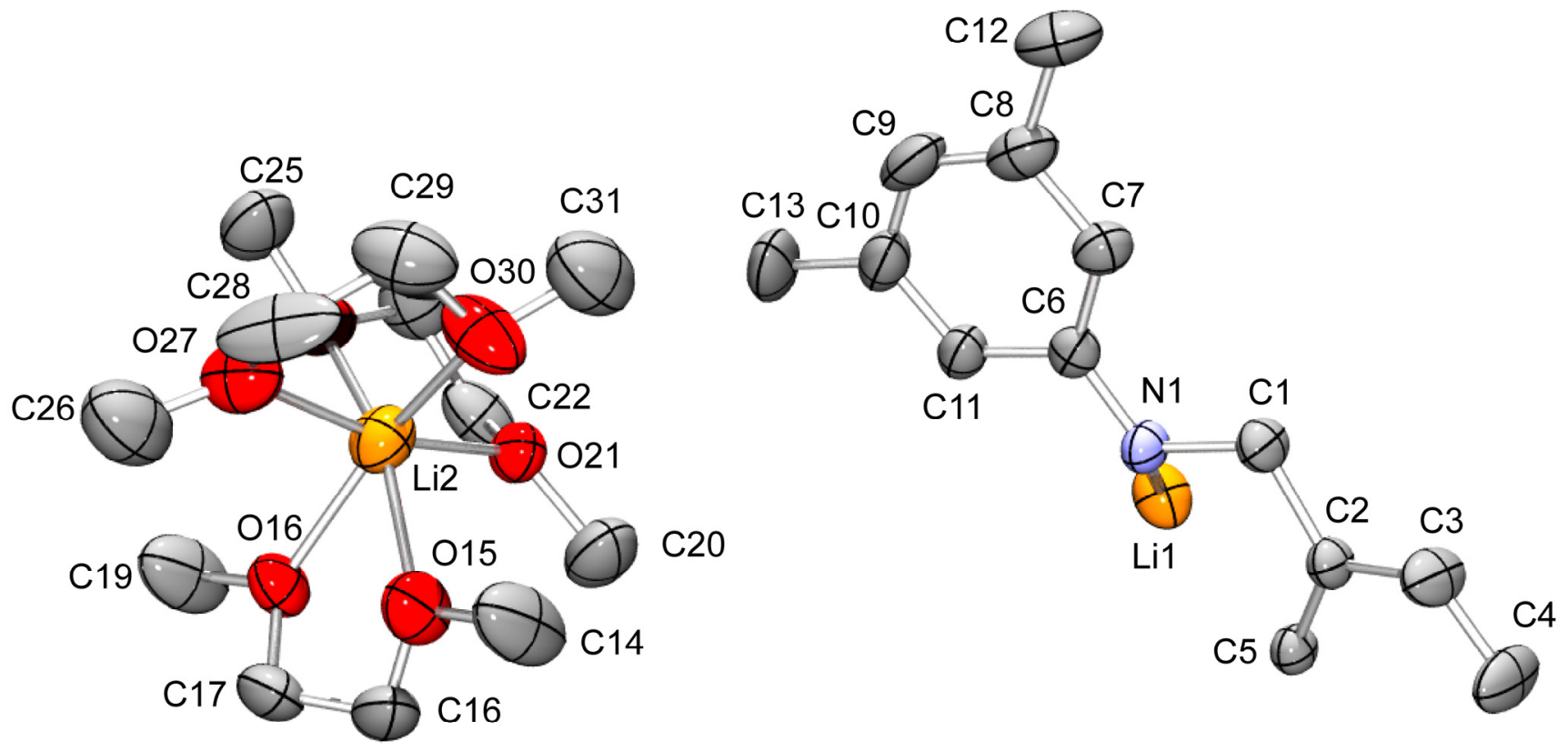

Figure S4. Asymmetric Unit for [3,5- $\left.\mathrm{MeNCNLi}_{2}\right]_{2}\left[\mathrm{Li}_{2}(\mathrm{DME})_{6}\right]$ (5) with ellipsoids drawn at the $50 \%$ probability level. Hydrogen atoms omitted for clarity. 


\section{$\mathrm{X}$-ray experimental details for $\left[(3,5-\mathrm{MeNCN})_{2} \mathrm{Hf}\right]\left[\mathrm{Li}_{2}(\mathrm{DME})_{2}\right](6)$ :}

Data were collected at $173 \mathrm{~K}$ on a Siemens SMART PLATFORM equipped with A CCD area detector and a graphite monochromator utilizing Mo $\mathrm{K}_{\alpha}$ radiation $(\lambda=0.71073 \AA)$. Cell parameters were refined using up to 8192 reflections. A full sphere of data (1850 frames) was collected using the $\omega$-scan method $\left(0.3^{\circ}\right.$ frame width). The first 50 frames were re-measured at the end of data collection to monitor instrument and crystal stability (maximum correction on I was $<1 \%$ ). Absorption corrections by integration were applied based on measured indexed crystal faces. The structure was solved by the Direct Methods in SHELXTL6, and refined using full-matrix least squares. The non-H atoms were treated anisotropically, whereas the hydrogen atoms were calculated in ideal positions and were riding on their respective carbon atoms. The asymmetric unit consists of two chemically equivalent but crystallographically independent complexes. A total of 1207 parameters were refined in the final cycle of refinement using 13830 reflections with $\mathrm{I}>2 \alpha(\mathrm{I})$ to yield $\mathrm{R}_{1}$ and $\mathrm{wR}_{2}$ of $4.22 \%$ and $6.60 \%$, respectively. Refinement was done using $\mathrm{F}^{2}$. 
Table S3. Crystal data, structure solution and refinement for $[(3,5-$

$\left.\mathrm{MeNCN})_{2} \mathrm{Hf}\right]\left[\mathrm{Li}_{2}(\mathrm{DME})_{2}\right](\mathbf{6})$.

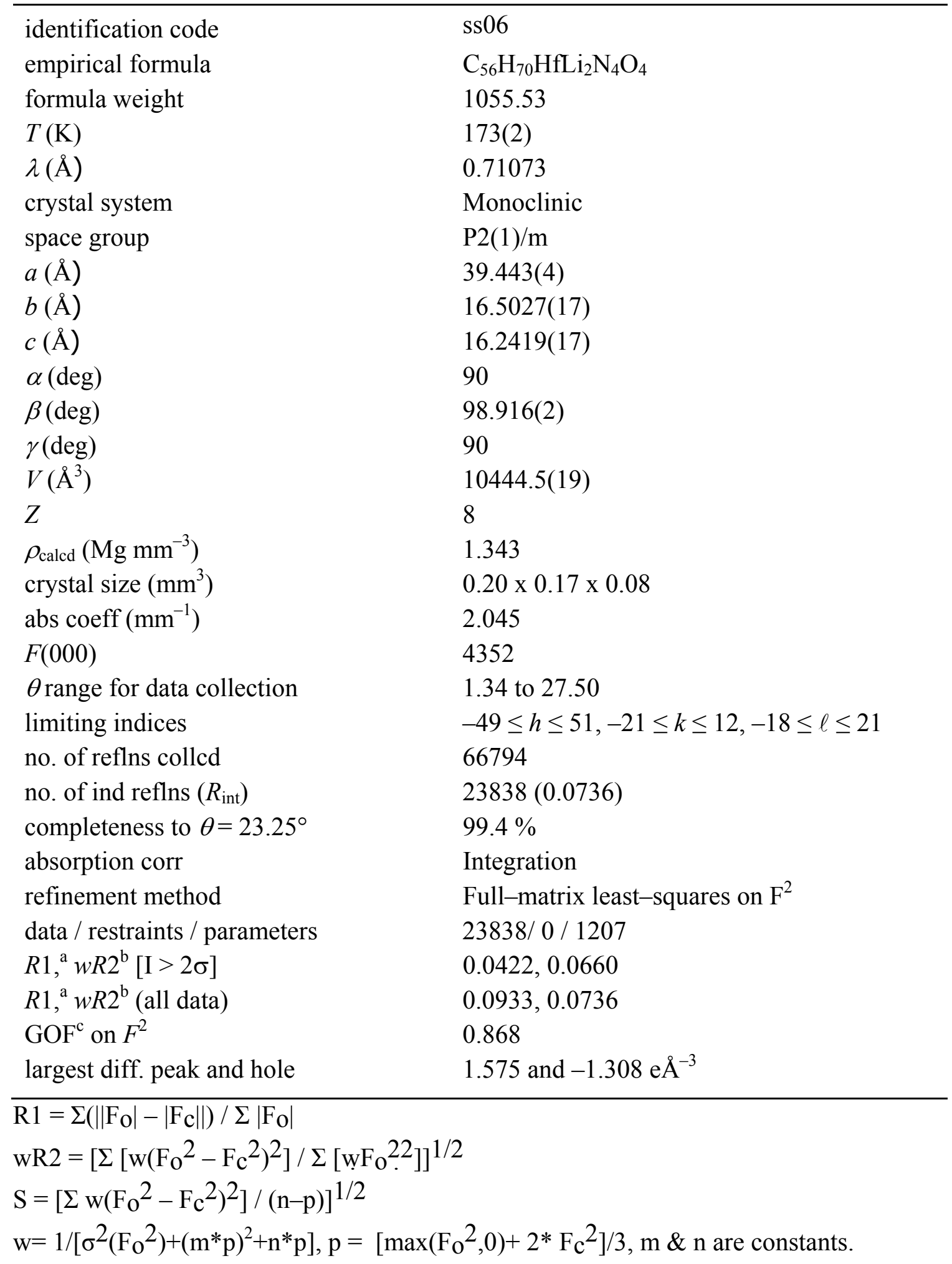




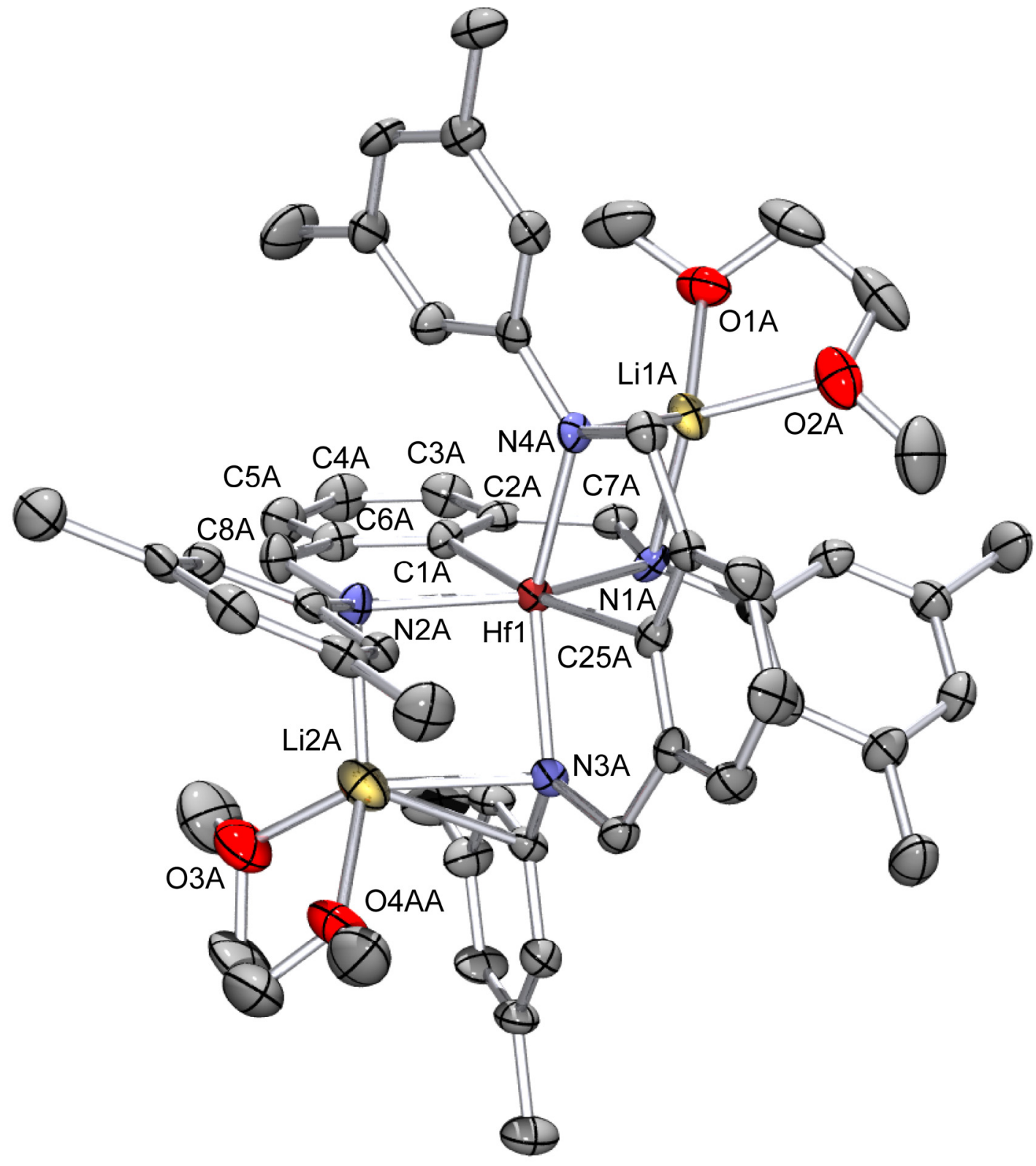

Figure S5. Molecular Structure of $\left[(3,5-\mathrm{MeNCN})_{2} \mathrm{Hf}\right]\left[\mathrm{Li}_{2}(\mathrm{DME})_{2}\right](6)$ with ellipsoids drawn at the $50 \%$ probability level. Hydrogen atoms, lithium counter ions and DME omitted for clarity. 


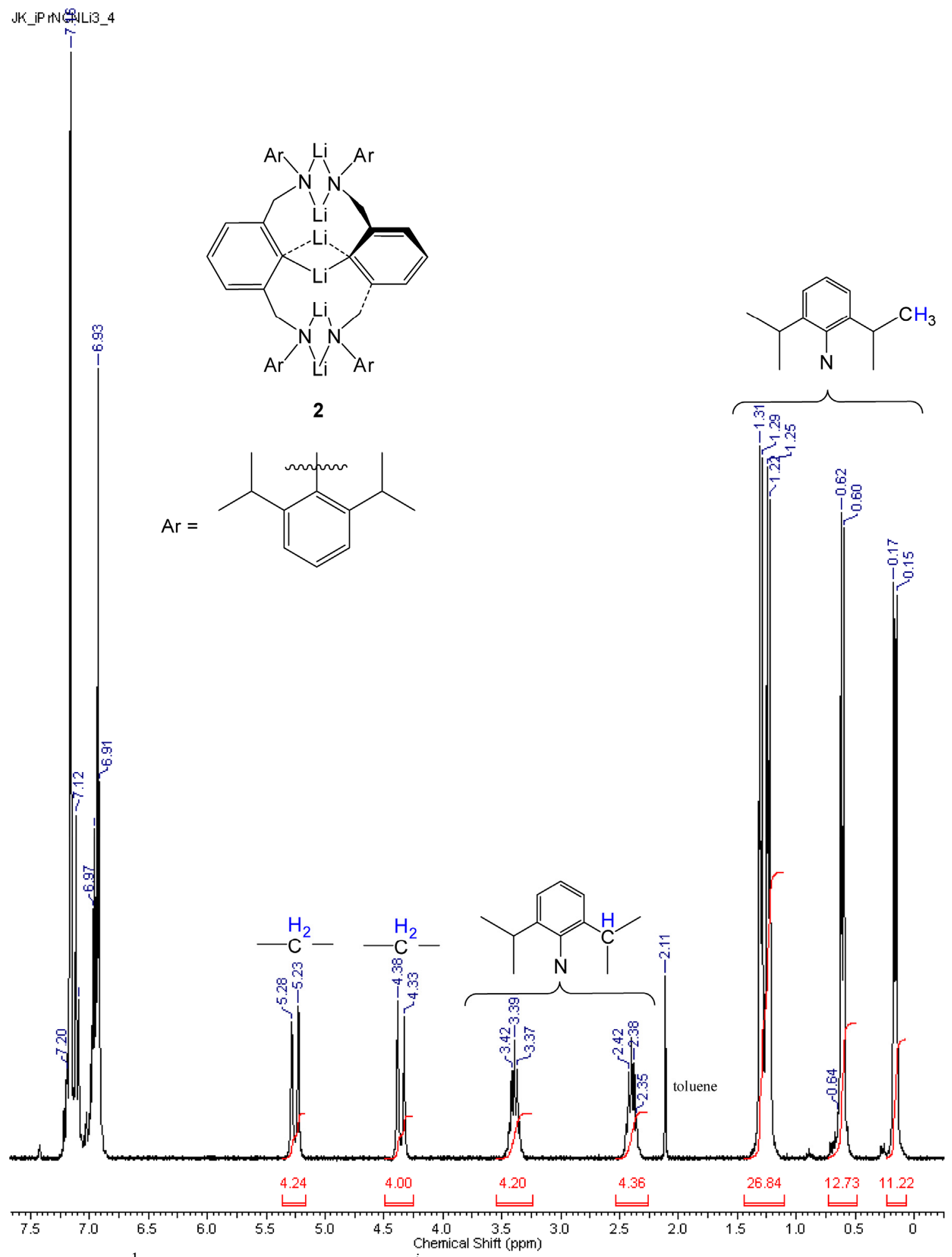

Figure S6. ${ }^{1} \mathrm{H}$ NMR spectrum of $\left[2,6-{ }^{i} \mathrm{PrNCNLi}_{3}\right]_{2}$ (2) in $\mathrm{C}_{6} \mathrm{D}_{6}(\mathrm{Ar}=2,6-$ diisopropylphenyl). 


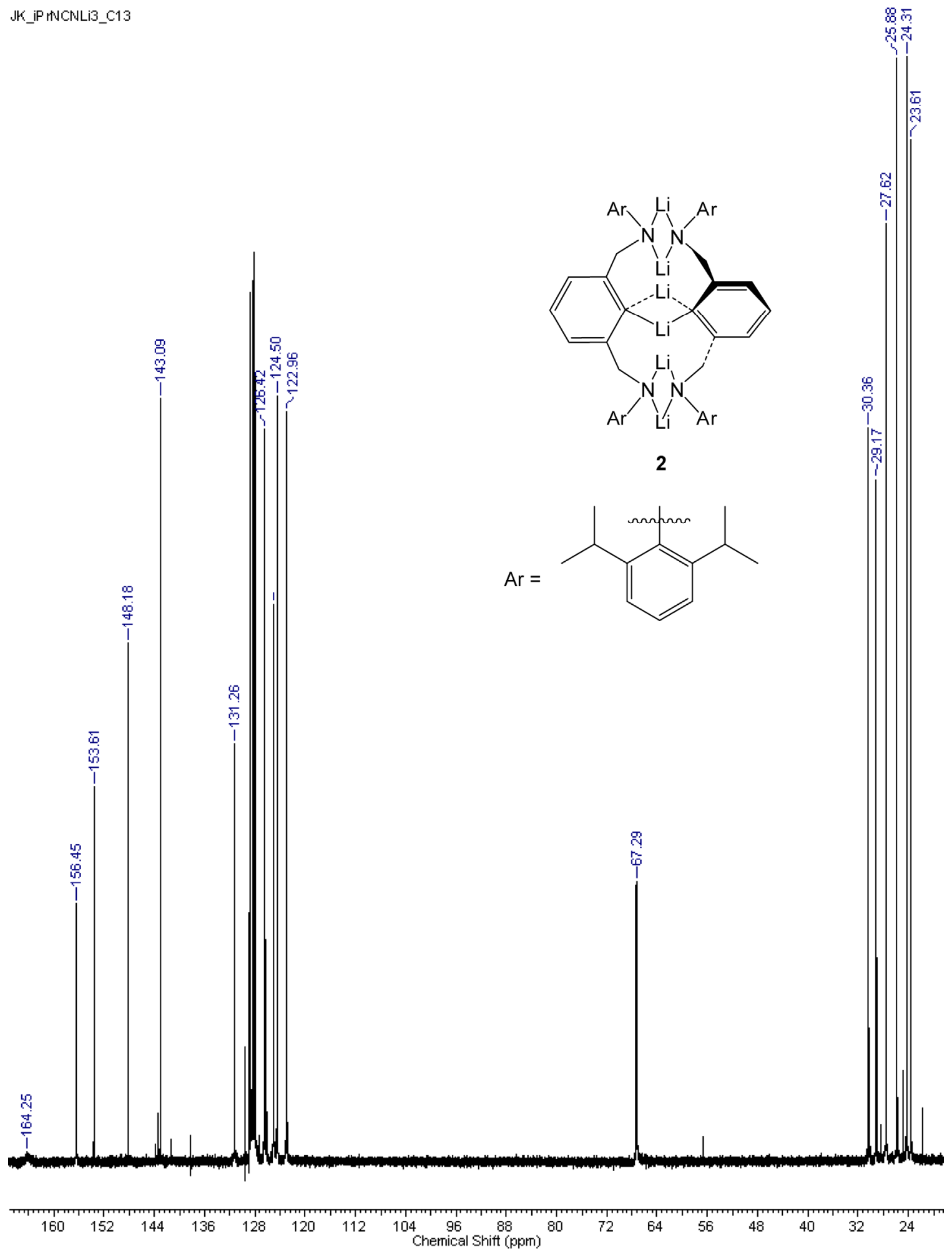

Figure S7. ${ }^{13} \mathrm{C}\left\{{ }^{1} \mathrm{H}\right\} \quad \mathrm{NMR}$ spectrum of $\left[2,6-{ }^{i} \mathrm{PrNCNLi}_{3}\right]_{2}$ (2) in $\mathrm{C}_{6} \mathrm{D}_{6} \quad(\mathrm{Ar}=2,6-$ diisopropylphenyl). 


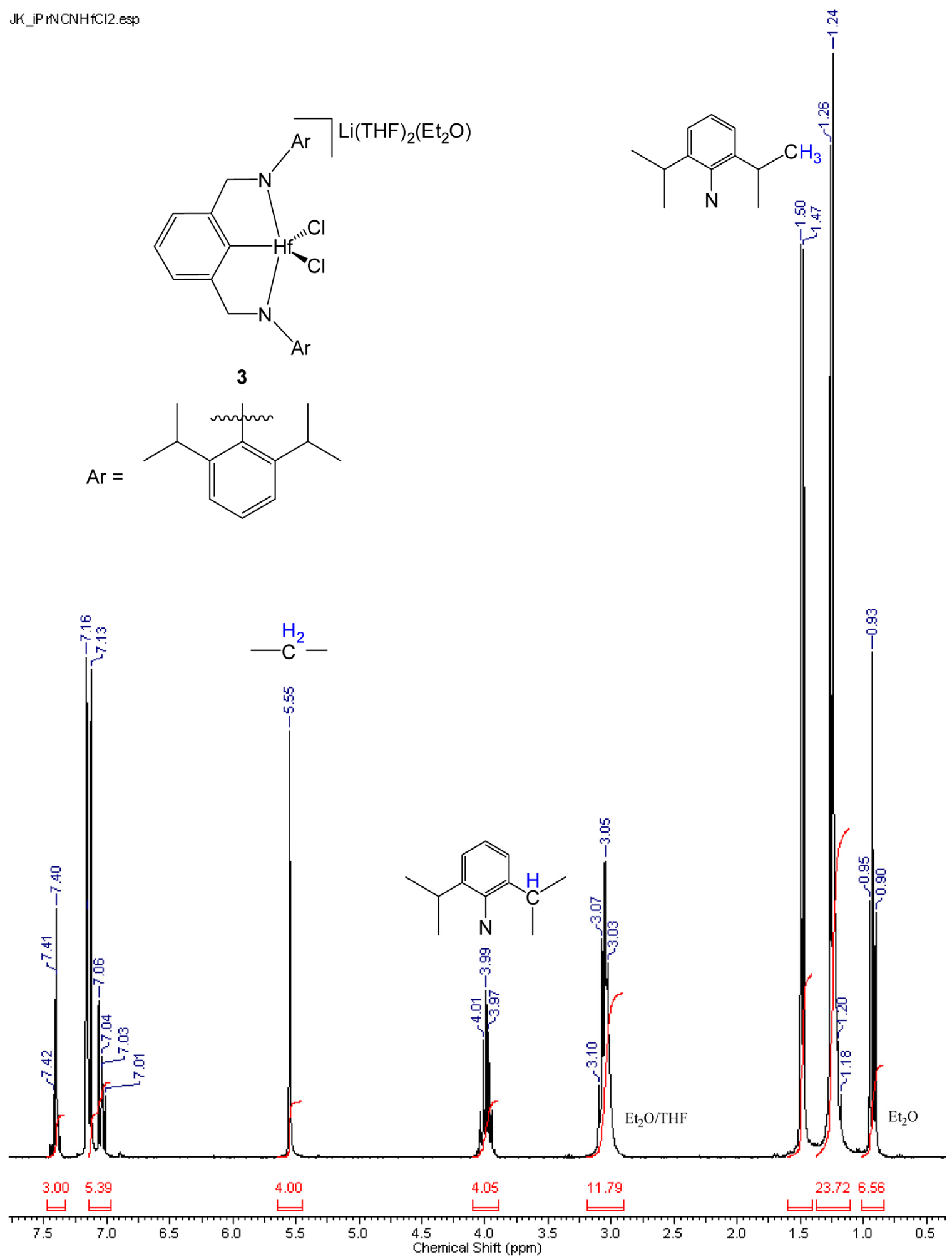

Figure S8. ${ }^{1} \mathrm{H}$ NMR spectrum of $\left[\left(2,6-{ }^{i} \mathrm{PrNCN} \mathrm{HfCl}_{2}\right]\left[\mathrm{Li}(\mathrm{THF})_{2}\left(\mathrm{Et}_{2} \mathrm{O}\right)\right](3)\right.$ in $\mathrm{C}_{6} \mathrm{D}_{6}(\mathrm{Ar}=2,6-$ diisopropylphenyl). 


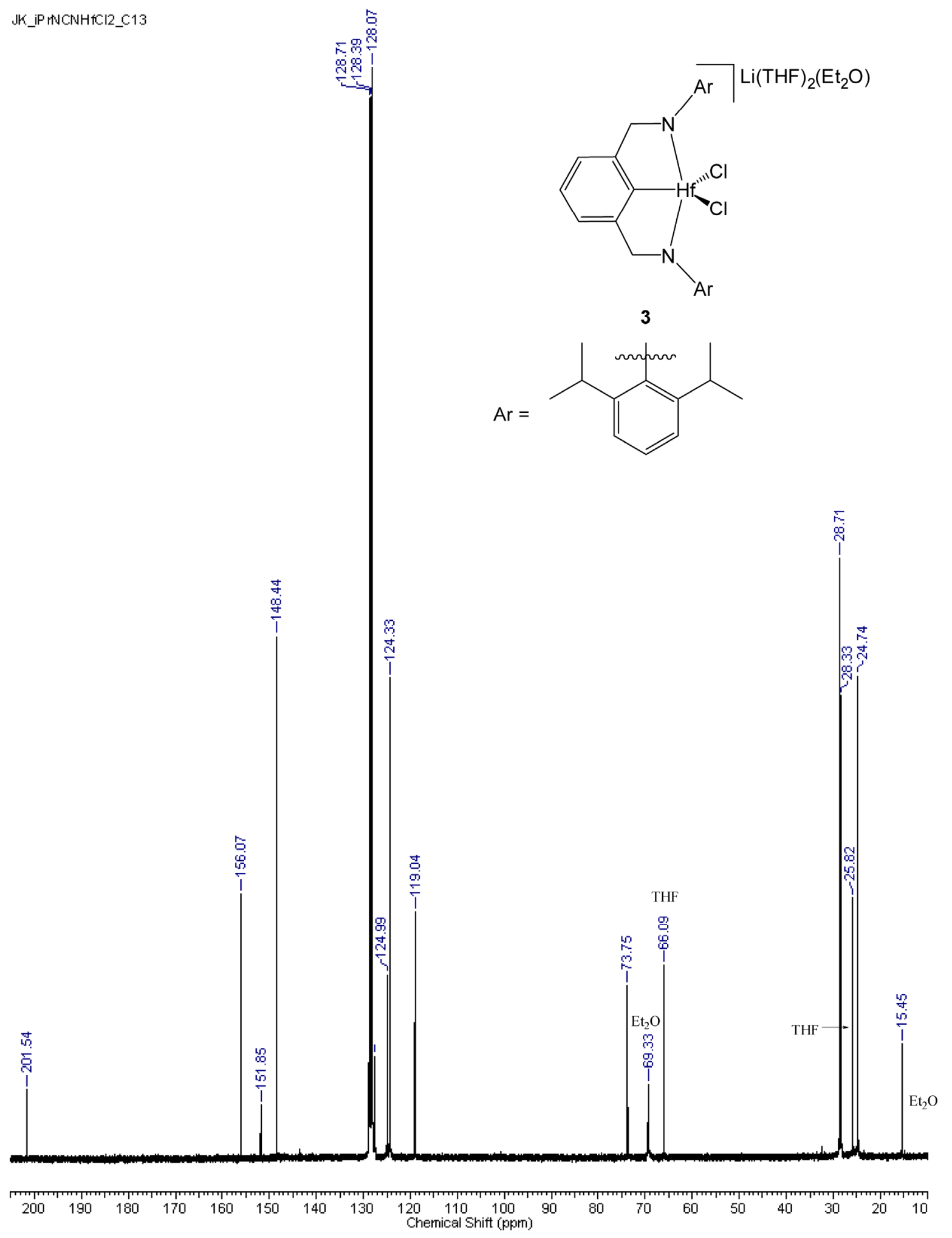

Figure S9. ${ }^{13} \mathrm{C}\left\{{ }^{1} \mathrm{H}\right\}$ NMR spectrum of $\left[\left(2,6-{ }^{i} \mathrm{PrNCN}\right) \mathrm{HfCl}_{2}\right]\left[\mathrm{Li}(\mathrm{THF})_{2}\left(\mathrm{Et}_{2} \mathrm{O}\right)\right](3)$ in $\mathrm{C}_{6} \mathrm{D}_{6}(\mathrm{Ar}=$ 2,6-diisopropylphenyl). 


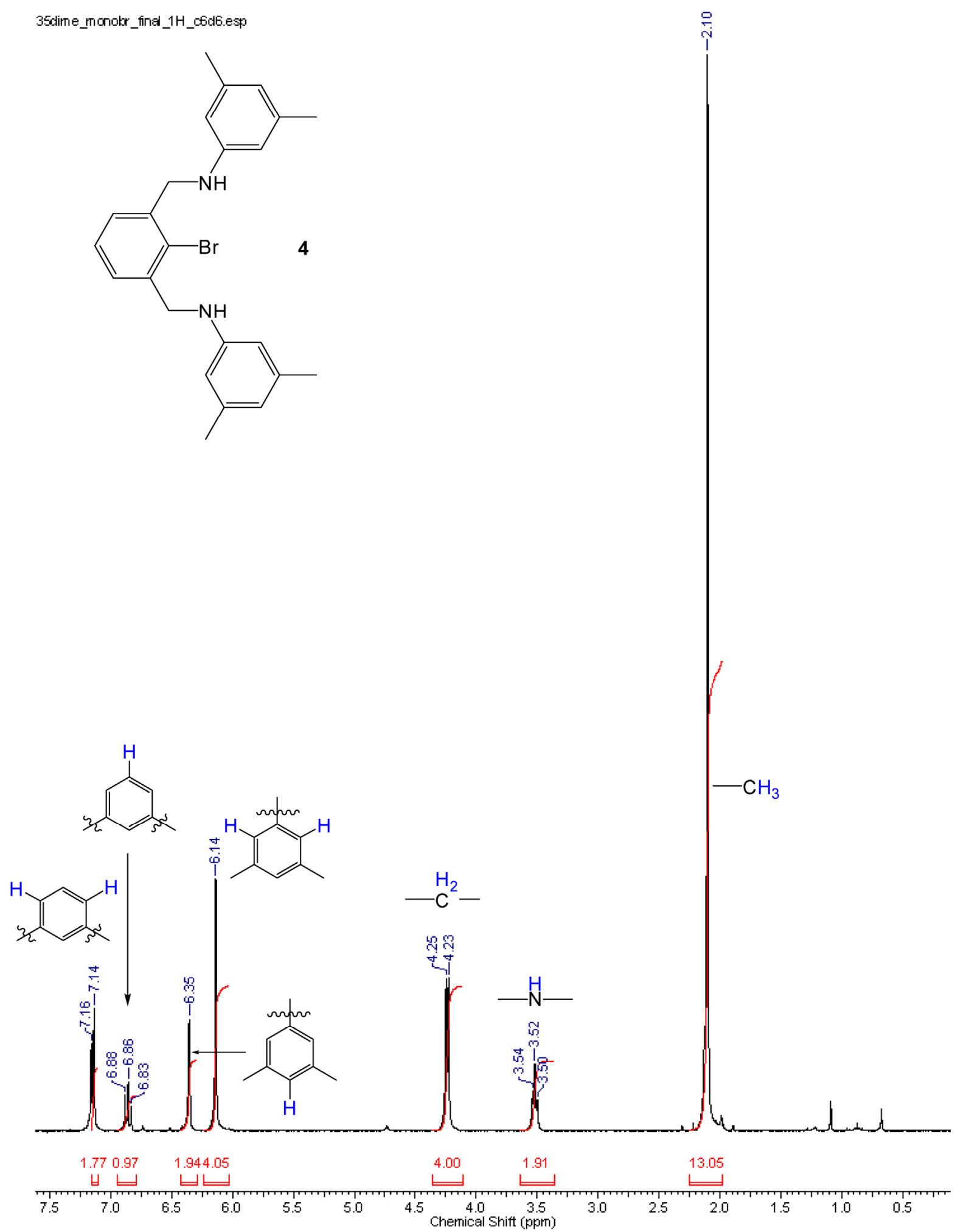

Figure S10. ${ }^{1} \mathrm{H}$ NMR spectrum of $3,5-\mathrm{MeNCNH}_{2} \mathrm{Br}(4)$ in $\mathrm{C}_{6} \mathrm{D}_{6}$. 


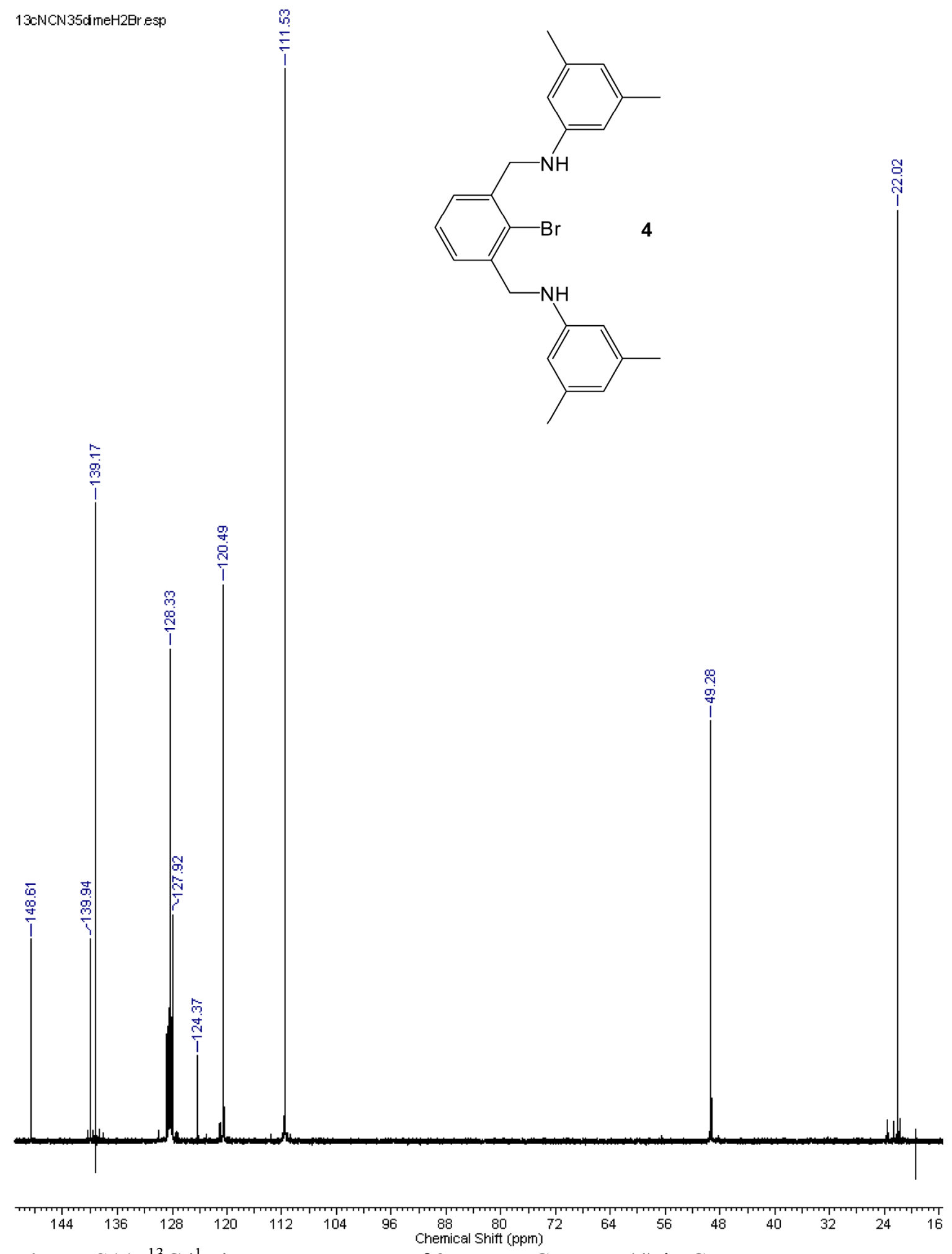

Figure S11. ${ }^{13} \mathrm{C}\left\{{ }^{1} \mathrm{H}\right\}$ NMR spectrum of 3,5-MeNCNH${ }_{2} \mathrm{Br}(4)$ in $\mathrm{C}_{6} \mathrm{D}_{6}$. 
35dime li3_1H_find
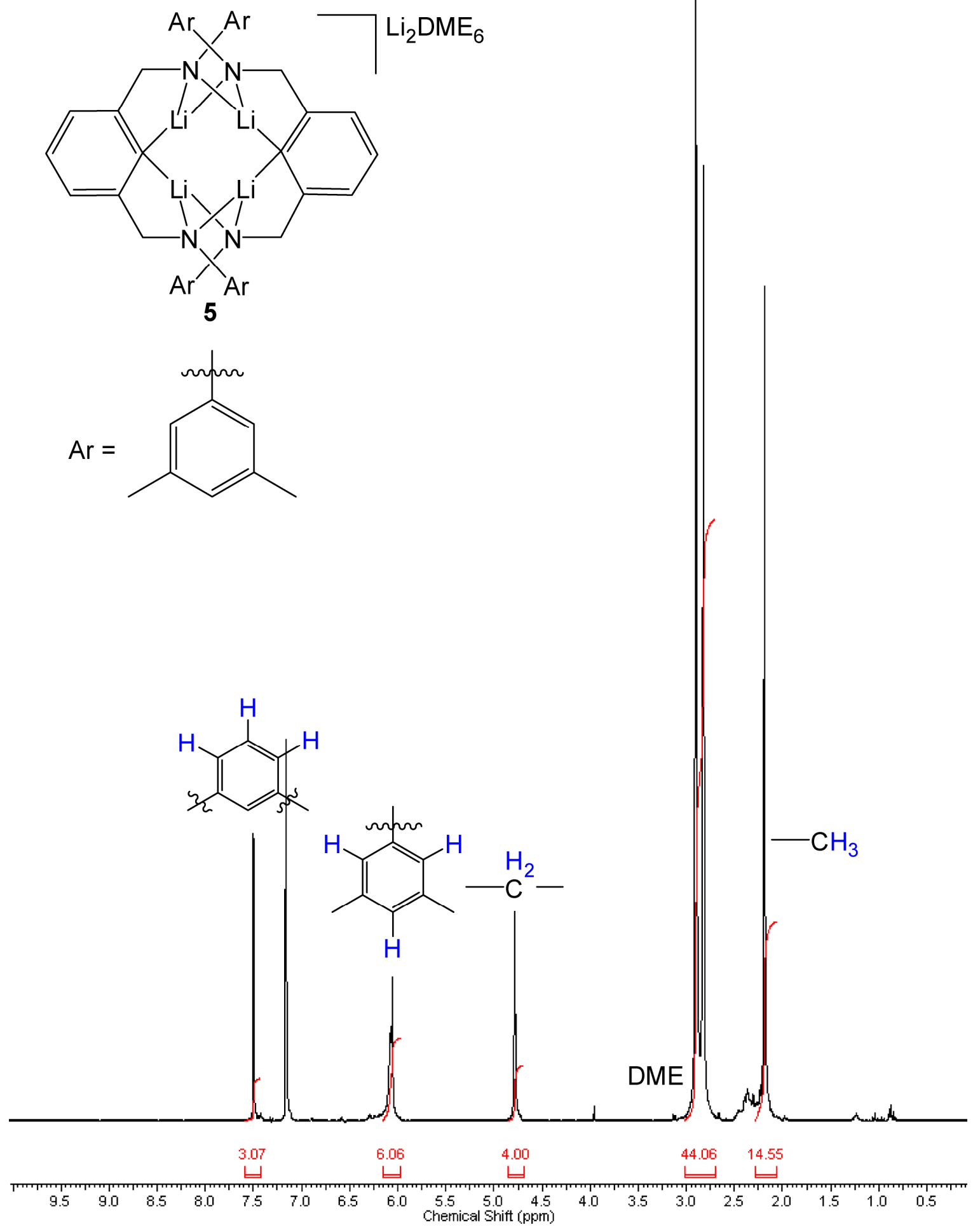

Figure S12. ${ }^{1} \mathrm{H}$ NMR spectrum of $\left[3,5-\mathrm{MeNCNLi}_{2}\right]_{2}\left[\mathrm{Li}_{2}(\mathrm{DME})_{6}\right](\mathbf{5})$ in $\mathrm{C}_{6} \mathrm{D}_{6}(\mathrm{Ar}=3,5-$ dimethylphenyl). 


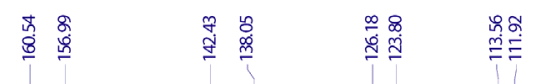

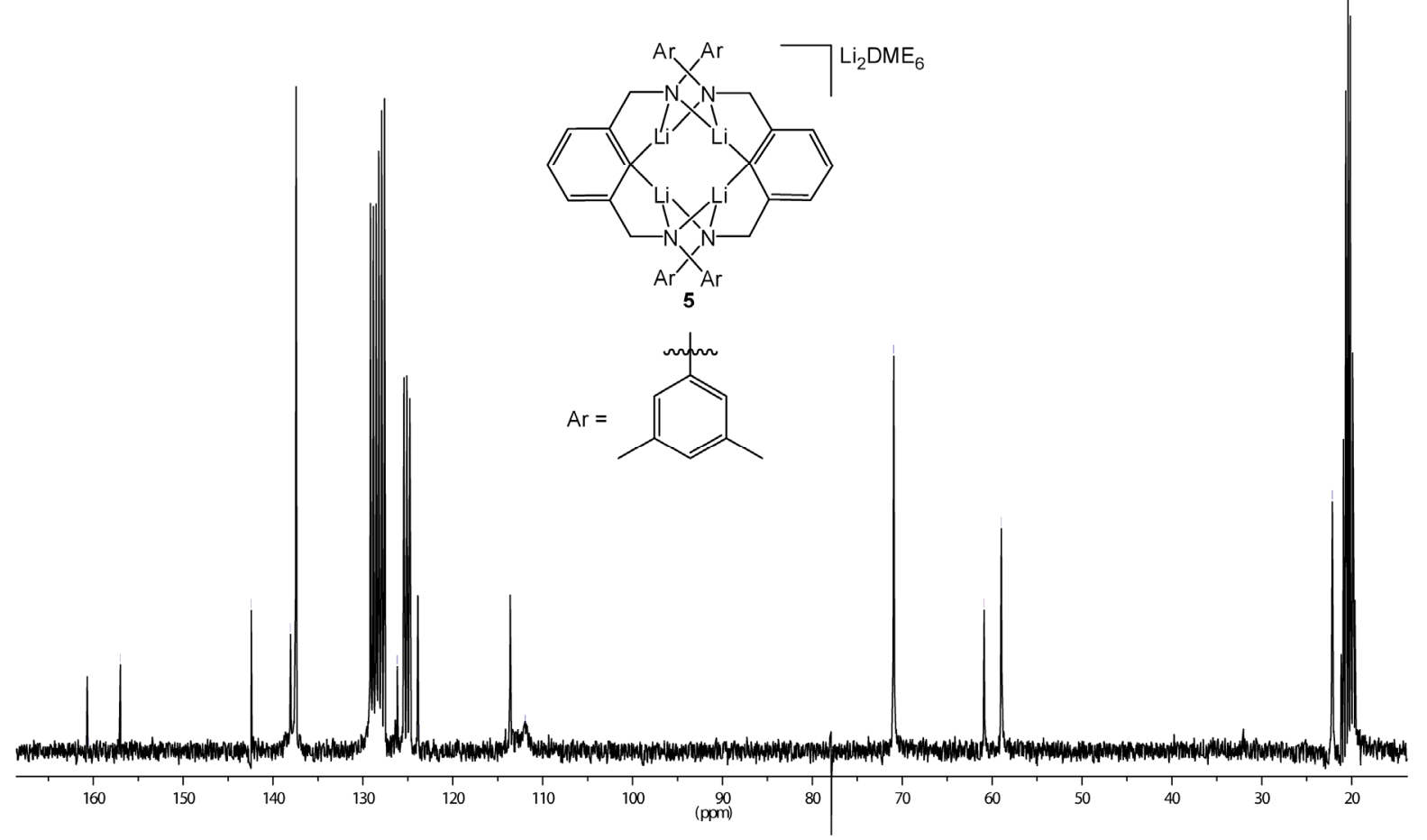

Figure S13. ${ }^{13} \mathrm{C}\left\{{ }^{1} \mathrm{H}\right\}$ NMR spectrum of $\left[3,5-\mathrm{MeNCNLi}_{2}\right]_{2}\left[\mathrm{Li}_{2}(\mathrm{DME})_{6}\right](\mathbf{5})$ in $\mathrm{C}_{6} \mathrm{D}_{6}(\mathrm{Ar}=3,5-$ dimethylphenyl). 

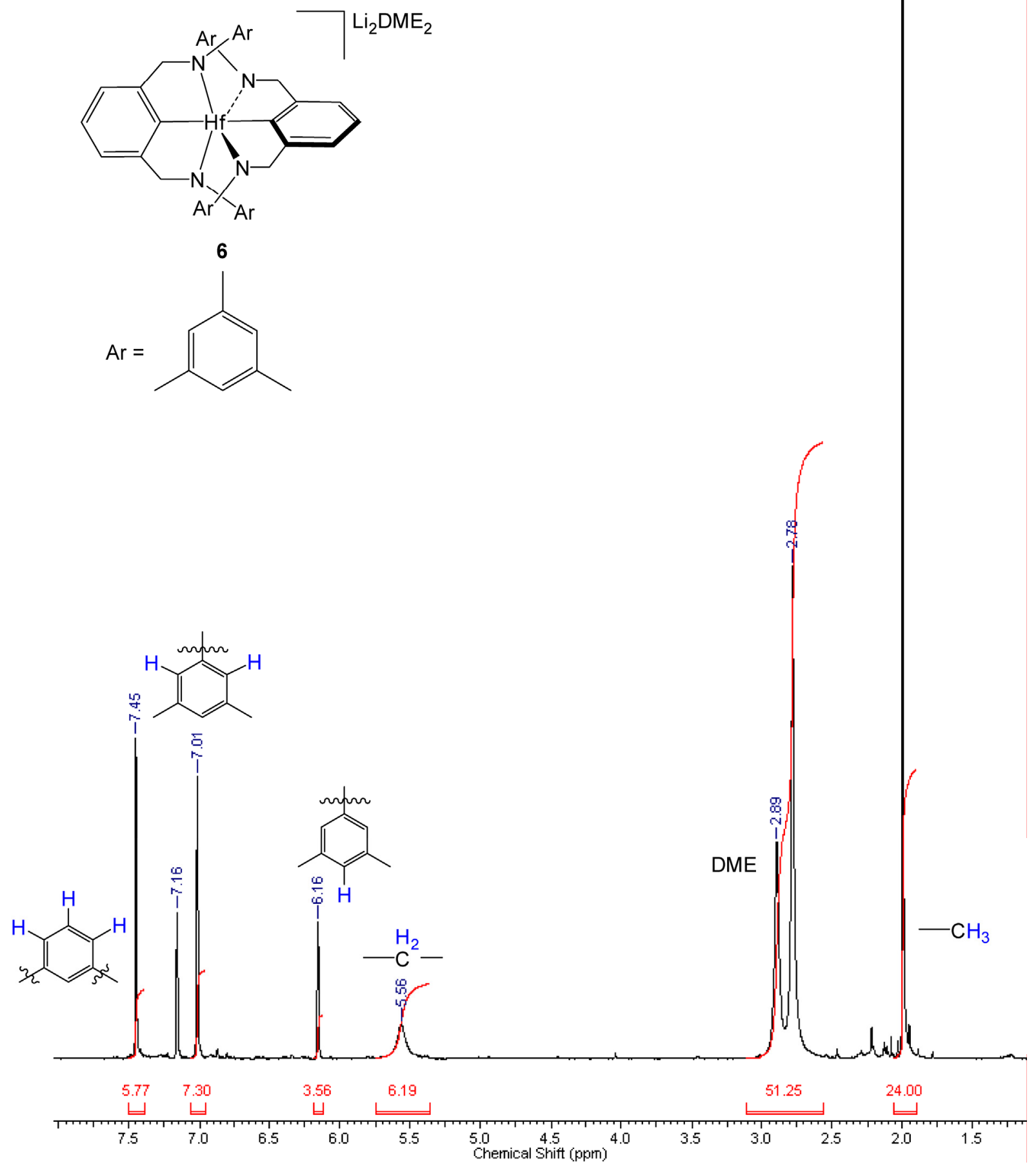

Figure S14. ${ }^{1} \mathrm{H}$ NMR spectrum of $\left[(3,5-\mathrm{MeNCN})_{2} \mathrm{Hf}\right]\left[\mathrm{Li}_{2}(\mathrm{DME})_{2}\right](6)$ in $\mathrm{C}_{6} \mathrm{D}_{6}(\mathrm{Ar}=3,5-$ dimethylphenyl). 


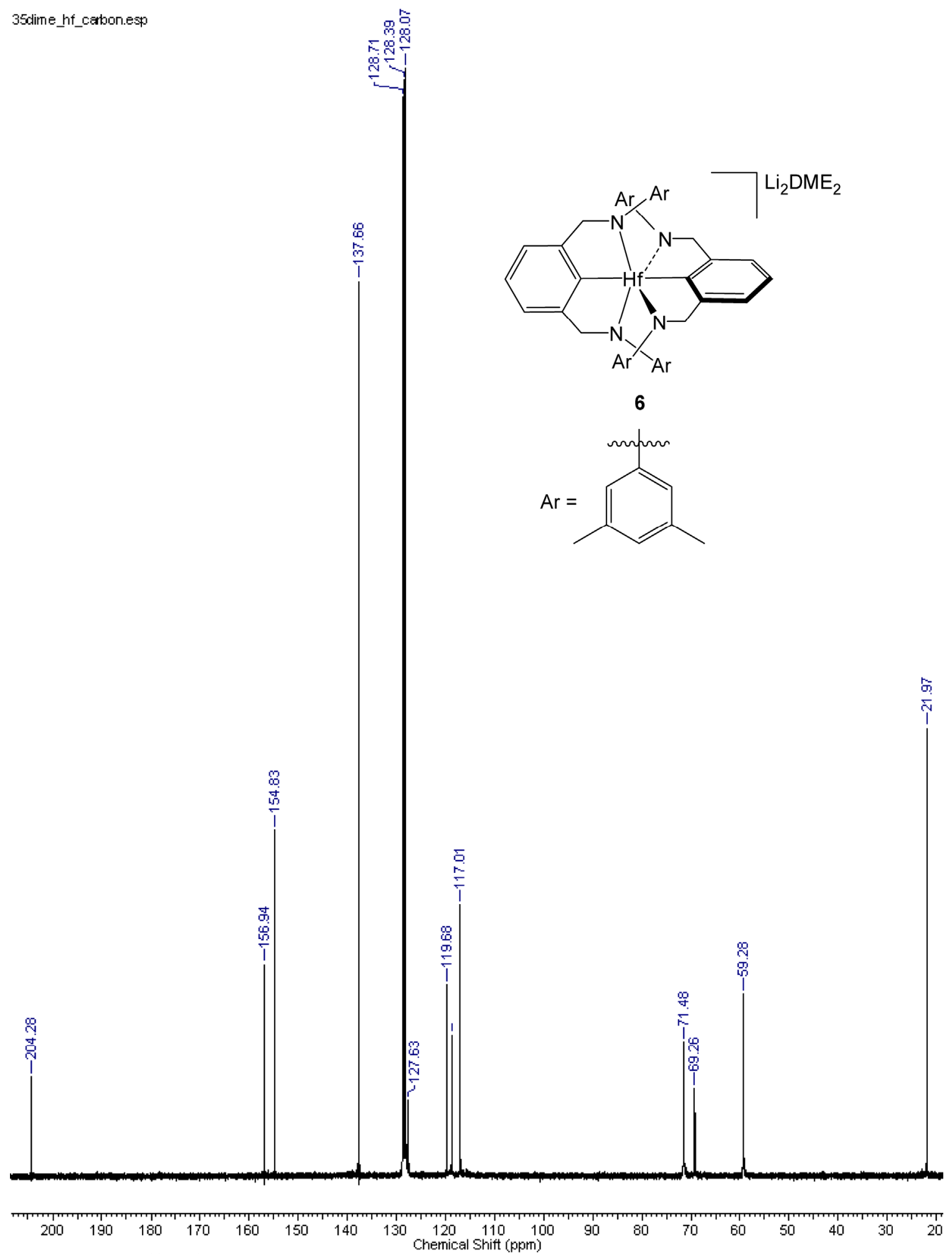

Figure S15. ${ }^{13} \mathrm{C}\left\{{ }^{1} \mathrm{H}\right\}$ NMR spectrum of $\left[(3,5-\mathrm{MeNCN})_{2} \mathrm{Hf}\right]\left[\mathrm{Li}_{2}(\mathrm{DME})_{2}\right](\mathbf{6})$ in $\mathrm{C}_{6} \mathrm{D}_{6}(\mathrm{Ar}=3,5-$ dimethylphenyl). 